Draft Version OCtober 25, 2018

Preprint typeset using $\mathrm{LAT}_{\mathrm{E} X} \mathrm{X}$ style emulateapj v. 08/22/09

\title{
GALAXY GROUPS IN THE SDSS DR4: III. THE LUMINOSITY AND STELLAR MASS FUNCTIONS
}

\author{
XiaOHU YANG ${ }^{1,4}$, H.J. Mo ${ }^{2}$, Frank C. VAN DEN BOSCH ${ }^{3}$ \\ Draft version October 25, 2018
}

\begin{abstract}
Using a large galaxy group catalogue constructed from the Sloan Digital Sky Survey Data Release 4 (SDSS DR4) with an adaptive halo-based group finder, we investigate the luminosity and stellar mass functions for different populations of galaxies (central versus satellite; red versus blue; and galaxies in groups of different masses) and for groups themselves. The conditional stellar mass function (CSMF), which describes the stellar distribution of galaxies in halos of a given mass for central and satellite galaxies can be well modeled with a log-normal distribution and a modified Schechter form, respectively. On average, there are about 3 times as many central galaxies as satellites. Among the satellite population, there are in general more red galaxies than blue ones. For the central population, the luminosity function is dominated by red galaxies at the massive end, and by blue galaxies at the low mass end. At the very low-mass end $\left(M_{*} \lesssim 10^{9} h^{-2} \mathrm{M}_{\odot}\right)$, however, there is a marked increase in the number of red centrals. We speculate that these galaxies are located close to large halos so that their star formation is truncated by the large-scale environments. The stellar-mass function of galaxy groups is well described by a double power law, with a characteristic stellar mass at $\sim 4 \times 10^{10} h^{-2} \mathrm{M}_{\odot}$. Finally, we use the observed stellar mass function of central galaxies to constrain the stellar mass halo mass relation for low mass halos, and obtain $M_{*, c} \propto M_{h}^{4.9}$ for $M_{h} \ll 10^{11} h^{-1} \mathrm{M}_{\odot}$.

Subject headings: dark matter - large-scale structure of the universe - galaxies: halos - methods: statistical
\end{abstract}

\section{INTRODUCTION}

In recent years, great progress has been made in our understanding about how galaxies form and evolve in dark matter halos owing to the development of halo models and the related halo occupation models. The halo occupation distribution (hereafter HOD), $P\left(N \mid M_{h}\right)$, which gives the probability of finding $N$ galaxies (with some specified properties) in a halo of mass $M_{h}$, has been extensively used to study the galaxy distribution in dark matter halos and galaxy clustering on large scales (e.g. Jing, Mo \& Börner 1998; Peacock \& Smith 2000; Seljak 2000; Scoccimarro et al. 2001; Jing, Börner \& Suto 2002; Berlind \& Weinberg 2002; Bullock, Wechsler \& Somerville 2002; Scranton 2002; Zehavi et al. 2004, 2005; Zheng et al. 2005; Tinker et al. 2005; Skibba et al. 2007; Brown et al. 2008). The conditional luminosity function (hereafter CLF), $\Phi\left(L \mid M_{h}\right) \mathrm{d} L$, which refines the HOD statistic by considering the average number of galaxies with luminosity $L \pm \mathrm{d} L / 2$ that reside in a halo of mass $M_{h}$, has also been investigated extensively (Yang, Mo \& van den Bosch 2003; van den Bosch, Yang \& Mo 2003; Vale \& Ostriker 2004, 2008; Cooray 2006; van den Bosch et al. 2007) and has been applied to various redshift surveys, such as the 2-degree Field Galaxy Redshift Survey (2dFGRS), the Sloan Digital Sky Survey (SDSS) and DEEP2 (e.g. Yan, Madgwick \& White 2003; Yang

\footnotetext{
${ }^{1}$ Shanghai Astronomical Observatory, the Partner Group of MPA, Nandan Road 80, Shanghai 200030, China; E-mail: xhyang@shao.ac.cn

2 Department of Astronomy, University of Massachusetts, Amherst MA 01003-9305

${ }^{3}$ Max-Planck-Institute for Astronomy, Königstuhl 17, D-69117 Heidelberg, Germany

${ }^{4}$ Joint Institute for Galaxy and Cosmology (JOINGC) of Shanghai Astronomical Observatory and University of Science and Technology of China
}

et al. 2004; Mo et al. 2004; Wang et al. 2004; Zehavi et al. 2005; Yan, White \& Coil 2004). These investigations demonstrate that the HOD/CLF statistics are very powerful tools to establish and describe the connection between galaxies and dark matter halos, providing important constraints on various physical processes that govern the formation and evolution of galaxies, such as gravitational instability, gas cooling, star formation, merging, tidal stripping and heating, and a variety of feedback processes, and how their efficiencies scale with halo mass. Furthermore, they also indicate that the galaxy/dark halo connection can provide important constraints on cosmology (e.g.,van den Bosch, Mo \& Yang 2003; Zheng \& Weinberg 2007).

However, as pointed out in Yang et al. (2005c; hereafter $\mathrm{Y05c}$ ), a shortcoming of the HOD/CLF models is that the results are not completely model independent. Typically, assumptions have to be made regarding the functional form of either $P\left(N \mid M_{h}\right)$ or $\Phi\left(L \mid M_{h}\right)$. Moreover, in all HOD/CLF studies to date, the occupation distributions have been determined in an indirect way: the free parameters of the assumed functional form are constrained using statistical data on the abundance and clustering properties of the galaxy population. An alternative method that can directly probe the galaxy dark halo connection (e.g. HOD/CLF models) is to use galaxy groups as a representation of dark matter halos and to study how the galaxy population changes with the properties of the groups (e.g., Y05c; Zandivarez et al. 2006; Robotham et al. 2006; Hansen et al. 2007; Yang et al. 2008). For such a purpose, one has to properly find the galaxy groups that are closely connected to the dark matter halos. In recent studies, Yang et al. (2005a; 2007) developed an adaptive halo-based group 
finder that has such features ${ }^{5}$. This group finder has been applied to the 2dFGRS (Yang et al. 2005a) and to the SDSS (Weinmann et al. 2006a; Yang et al. 2007). Detailed tests with mock galaxy catalogues have shown that this group finder is very successful in associating galaxies according to their common dark matter halos. In particular, the group finder performs reliably not only for rich systems, but also for poor systems, including isolated central galaxies in low mass halos. This makes it possible to study the galaxy-halo connection for systems covering a large dynamic range in masses. With a welldefined galaxy group catalogue, one can then not only study the properties of galaxies in different groups (e.g. Y05c; Yang et al. 2005d; Collister \& Lahav 2005; van den Bosch et al. 2005; Robotham et al. 2006; Zandivarez et al. 2006; Weinmann et al. 2006a,b; van den Bosch et al. 2008; McIntosh et al. 2007; Yang et al. 2008), but also probe how dark matter halos trace the large-scale structure of the Universe (e.g. Yang et al. 2005b, 2006; Coil et al. 2006; Berlind et al. 2007; Wang et al. 2008a).

Recently, this group finder has been applied to the Sloan Digital Sky Survey Data Release 4 (SDSS DR4), and the group catalogues constructed are described in detail in Yang et al. (2007; Paper I hereafter). In these catalogues various observational selection effects are taken into account, and each of the groups is assigned a reliable halo mass. The group catalogues including the membership of the groups are available at these links ${ }^{6}$ 7. In Yang et al. (2008; Paper II hereafter) we have used these group catalogues to obtain various halo occupation statistics and to measure the CLFs for different populations of galaxies. In this paper, the third in the series, we will focus on the conditional stellar mass functions (CSMFs) for different populations of galaxies. In addition, we will also examine the general luminosity and stellar mass functions for different populations of galaxies and for groups themselves. Finally, we will demonstrate how to use the observed luminosity and stellar mass functions for central galaxies to constrain the HOD in small halos.

This paper is organized as follows: In Section 2 we describe the data (galaxy and group catalogues) used in this paper. Section 4 presents our measurement of the CSMFs for all, red and blue galaxies. Sections 3 and [5] present our measurement of the luminosity and stellar mass functions for galaxies and groups, respectively. In Section 6, we probe the properties of the central galaxies that can be formed in those small halos. Finally, we summarize our results in Section 7. Throughout this paper, we use a $\Lambda$ CDM 'concordance' cosmology whose parameters are consistent with the three-year data release of the WMAP mission: $\Omega_{m}=0.238, \Omega_{\Lambda}=0.762$, $n_{s}=0.951, h=0.73$ and $\sigma_{8}=0.75$ (Spergel et al. 2007). If not quoted, the units of luminosity, stellar and halo masses are in terms of $h^{-2} \mathrm{~L}_{\odot}, h^{-2} \mathrm{M}_{\odot}$ and $h^{-1} \mathrm{M}_{\odot}$, respectively. Finally, unless noted differently, the luminosity functions and stellar mass functions are presented in units of $h^{3} \mathrm{Mpc}^{-3} \mathrm{~d} \log L$ and $h^{3} \mathrm{Mpc}^{-3} \mathrm{~d} \log M_{*}$, respec-

\footnotetext{
${ }^{5}$ In this paper, we refer to systems of galaxies as groups regardless of their richness, including isolated galaxies (i.e., systems with a single member) and rich clusters of galaxies.

${ }^{6}$ http://gax.shao.ac.cn/data/Group.html

7 http://www.astro.umass.edu/ xhyang/Group.html
}

tively, where $\log$ is the 10 based logarithm.

\section{DATA}

\subsection{Galaxy and group catalogues}

The data used in our analysis here are the same as those used in Paper II. Readers who have already read through Paper II may go directly to the next subsection.

The group catalogues are constructed from the New York University Value-Added Galaxy Catalogue (NYUVAGC; Blanton et al. 2005b), which is based on the SDSS Data Release 4 (Adelman-McCarthy et al. 2006), but with an independent set of significantly improved reductions. From NYU-VAGC we select all galaxies in the Main Galaxy Sample with redshifts in the range $0.01 \leq z \leq 0.20$ and with a redshift completeness $\mathcal{C}>0.7$. As described in Paper I, three group samples are constructed from the corresponding galaxy samples: Sample I, which only uses the 362356 galaxies with measured $r$-band magnitudes and redshifts from the SDSS, Sample II which also includes 7091 galaxies with SDSS $r$-band magnitudes but redshifts taken from alternative surveys, and Sample III which includes an additional 38672 galaxies that lack redshifts due to fiber collisions but that are assigned the redshifts of their nearest neighbors. Although this fiber collision correction works well in roughly 60 percent of all cases, the remaining 40 percent are assigned redshifts that can be very different from their true values (Zehavi et al. 2002). Samples II and III should therefore be considered as two extremes as far as a treatment of fiber-collisions is concerned. Unless stated otherwise, our results are based on Sample II. For comparison, we also present some results obtained from Sample III.

The magnitudes and colors of all galaxies are based on the standard SDSS Petrosian technique (Petrosian 1976; Strauss et al. 2002), have been corrected for galactic extinction (Schlegel, Finkbeiner \& Davis 1998), and have been $K$-corrected and evolution corrected to $z=0.1$, using the method described in Blanton et al. (2003a; b). We use the notation ${ }^{0.1} \mathrm{M}_{r}-5 \log h$ to indicate the resulting absolute magnitude in the $r$-band. The galaxies are separated into red and blue subsamples according to their bi-normal distribution in the ${ }^{0.1}(g-r)$ color (Baldry et al. 2004; Blanton et al. 2005a; Li et al. 2006), using the separation criteria (see Paper II),

$$
{ }^{0.1}(g-r)=1.022-0.0651 x-0.00311 x^{2},
$$

where $x={ }^{0.1} \mathrm{M}_{r}-5 \log h+23.0$.

Stellar masses, indicated by $M_{*}$, for all galaxies are computed using the relations between stellar mass-tolight ratio and ${ }^{0.0}(g-r)$ color from Bell et al. (2003),

$$
\begin{aligned}
\log \left[\frac{M_{*}}{h^{-2} \mathrm{M}_{\odot}}\right]= & -0.306+1.097\left[{ }^{0.0}(g-r)\right]-0.10 \\
& -0.4\left({ }^{0.0} \mathrm{M}_{r}-5 \log h-4.64\right) .
\end{aligned}
$$

Here ${ }^{0.0}(g-r)$ and ${ }^{0.0} \mathrm{M}_{r}-5 \log h$ are the $(g-r)$ color and $r$-band magnitude $K+E$ corrected to $z=0.0 ; 4.64$ is the $r$-band magnitude of the Sun in the AB system (Blanton \& Roweis 2007); and the -0.10 term effectively implies that we adopt a Kroupa (2001) IMF (Borch et al. 2006). 
For each group in our catalogue we have two estimates of its dark matter halo mass $M_{h}$ : (1) $M_{L}$, which is based on the ranking of the characteristic group luminosity $L_{19.5}$, and (2) $M_{S}$, which is based on the ranking of the characteristic group stellar mass $M_{\text {stellar, }}$ respectively ${ }^{8}$. The halo mass is estimated for each group with at least one member galaxy that is brighter than ${ }^{0.1} \mathrm{M}_{r}-5 \log h=-19.5$. As shown in Paper I, these two halo masses agree reasonably well with each other, with scatter that decreases from $\sim 0.1$ dex at the low-mass end to $\sim 0.05$ dex at the massive end. Detailed tests using mock galaxy redshift surveys have demonstrated that the group masses thus estimated can recover the true halo masses with a 1- $\sigma$ deviation of $\sim 0.3 \mathrm{dex}$, and are more reliable than those based on the velocity dispersion of group members (Y05c; Weinmann et al. 2006; Berlind et al. 2006; Paper I). Note also that survey edge effects have been taken into account in our group catalogue: groups that suffer severely from edge effects (about 1.6\% of the total) have been removed from the catalogue. In most cases, we take the most massive galaxy (in terms of stellar mass) in a group as the central galaxy (MCG) and all others as satellite galaxies. In addition, we also considered a case in which the brightest galaxy in the group is considered as the central galaxy (BCG). Tests have shown that for most of what follows, these two definitions yield indistinguishable results. Whenever the two definitions lead to significant differences, we present results for both. Throughout this paper, results are calculated for both samples II and III using both halo masses, $M_{L}$ and $M_{S}$. Any significant differences in the results due to the use of different samples and mass estimates are discussed.

Finally, we caution that the SDSS pipeline may have underestimated the luminosities for bright galaxies (e.g. von der Linden et al. 2007; Guo et al. 2009). According to Guo et al. the NYU-VAGC magnitude is overestimated by about $0.5 \pm 0.1$ at apparent magnitudes $r \sim 13.0$ and about $0.1 \pm 0.1$ at $r \sim 17.0$. Although this will not change the halo masses estimated using the abundance match to halo mass function, the luminosity and stellar mass functions for galaxies and groups are shifted slightly at the bright ends if a correction is made to the SDSS luminosities.

\subsection{Galaxy and group completeness limits}

Because of the survey magnitude limit, only bright galaxies can be observed. This consequently will induce incompleteness in the distribution of galaxies with respect to absolute magnitude and stellar mass, and in the distribution of groups with halo mass. In this subsection, we discuss such completeness and how to make corrections.

As discussed in detail in the Appendix of van den Bosch et al. (2008), the apparent magnitude limit of the galaxy sample $\left(m_{r}=17.77\right)$ can be translated into a redshiftdependent absolute magnitude limit given by

$$
\begin{aligned}
& { }^{0.1} \mathrm{M}_{r, \lim }-5 \log h= \\
& 17.77-\operatorname{DM}(z)-k_{0.1}(z)+1.62(z-0.1) .
\end{aligned}
$$

${ }^{8} L_{19.5}$ and $M_{\text {stellar }}$ are, respectively, the total luminosity and total stellar mass of all group members with ${ }^{0.1} \mathrm{M}_{r}-5 \log h \leq$ -19.5 .

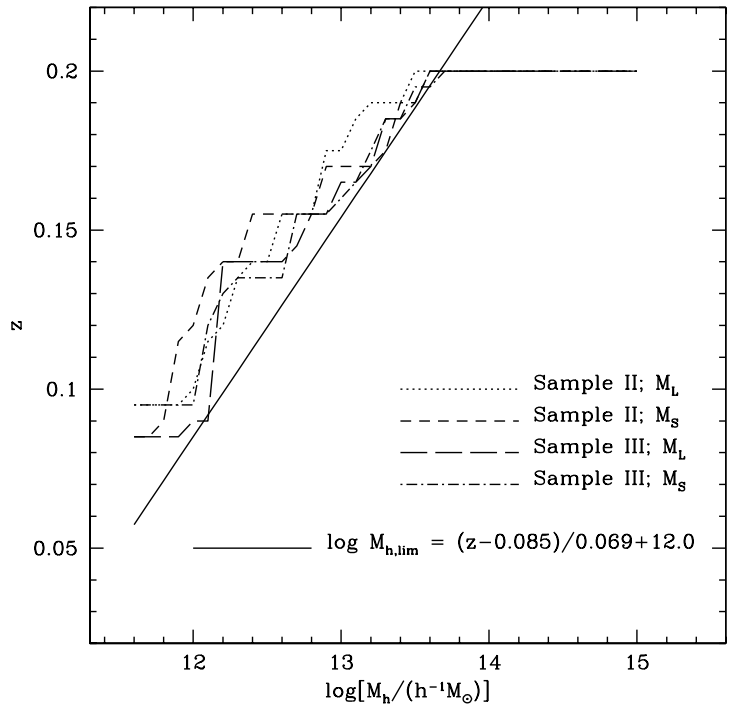

FIG. 1. - The group completeness limit as a function of halo mass. Different lines (except the solid one) correspond to different combinations of Samples and halo masses estimated $\left(M_{L}\right.$ or $\left.M_{S}\right)$ as indicated. The solid line illustrates a conservative halo mass limit for all those combinations. That is the halos below given redshift with halo masses larger than $M_{h, \text { lim }}$ are complete.

where $k_{0.1}(z)$ is the $K$-correction to $z=0.1$, the $1.62(z-0.1)$ term is the evolution correction of Blanton et al. (2003b), and

$$
\operatorname{DM}(z)=5 \log D_{L}(z)+25
$$

is the distance module corresponding to redshift $z$, with $D_{L}(z)$ the luminosity distance in $h^{-1} \mathrm{Mpc}$. Using the $K$-corrections of Blanton et al. (2003a; see also Blanton \& Roweis 2007), the redshift-dependence is reasonably well described by

$$
k_{0.1}(z)=2.5 \log \left(\frac{z+0.9}{1.1}\right) .
$$

At $z=0.1$, where (by definition) $k_{0.1}=-2.5 \log (1.1) \simeq$ -0.1 for all galaxies (e.g., Blanton \& Roweis 2007), this exactly gives the absolute magnitude limit of the sample. At lower and higher redshifts, however, a small fraction of the sample galaxies fall below this limit. This owes to the fact that $k_{0.1}(z)$ not only depends on redshift but also on color. In order to ensure completeness, we use a conservative absolute-magnitude limit:

$$
{ }^{0.1} \mathrm{M}_{r, \lim }^{\prime}-5 \log h={ }^{0.1} \mathrm{M}_{r, \lim }-5 \log h-0.1,
$$

where the term 0.1 takes into account the scatter in the $K$ correction. For the stellar masses of the SDSS galaxies, we adopt, for given redshift $z$, the following completeness limit:

$$
\begin{aligned}
& \log \left[M_{*, \lim } /\left(h^{-2} \mathrm{M}_{\odot}\right)\right]= \\
& \frac{4.852+2.246 \log D_{L}(z)+1.123 \log (1+z)-1.186 z}{1-0.067 z} .
\end{aligned}
$$

(see van den Bosch et al. 2008)

Next we consider incompleteness in the actual group catalogue. As illustrated in Fig. 6 of Yang et al. (2007), the groups within a certain luminosity $L_{19.5}$ or stellar mass $M_{\text {stellar }}$ bin (which corresponds to a certain halo 
mass bin) are complete only to a certain redshift. Beyond this redshift the number density of the groups drops dramatically. We therefore need to take this completeness into account. Here we proceed as follows to obtain the halo-mass completeness limit at any given redshift. First, for a given halo mass $M_{h}$, we measure the number densities of groups with halo mass within $\log M_{h} \pm 0.05$, $n\left(0.01, z_{\max }\right)$ and $n\left(z_{\max }-\Delta z, z_{\max }\right)$, within the redshift ranges, $\left[0.01, z_{\max }\right]$ and $\left[z_{\max }-\Delta z, z_{\max }\right]$, respectively. Here we set $\Delta z=0.005$. If the group sample is not complete at redshift $z_{\max }$, then the group number density $n\left(z_{\max }-\Delta z, z_{\max }\right)$ is expected to drop significantly. Starting from $z_{\max }=0.2$, we iteratively decrease $z_{\max }$ according to $z_{\max }=z_{\max }-\Delta z$, and find the largest redshift $z_{\max }$ that satisfies,

$$
n\left(z_{\max }-\Delta z, z_{\max }\right)+3 \sigma_{n} \geq n\left(0.01, z_{\max }\right)
$$

where $\sigma_{n}$ is the variance in the number density of groups within $\left[z_{\max }-\Delta z, z_{\max }\right]$ among 200 bootstrap samples. The $z_{\max }$ thus obtained is the one at which the halos are complete down to the corresponding halo mass $M_{h}$. For the group samples used in this paper, Samples II and III, and for the two sets of halo masses estimated for our groups, $M_{L}$ and $M_{S}$, we obtain the value of $z_{\max }$ as a function of halo mass. The results are shown in Fig. 1. From this plot, we obtain a conservative halo-mass limit,

$$
\log M_{h, \lim }=(z-0.085) / 0.069+12,
$$

which is shown as the solid line in Fig. 1. Clearly, this criterion works for both Samples II and III, as well as for both $M_{L}$ and $M_{S}$. Thus, for a given redshift $z$, groups with masses $\geq M_{h, \text { lim }}$ are complete.

\section{THE GALAXY LUMINOSITY AND STELLAR MASS FUNCTIONS: CENTRAL VS SATELLITE}

In this section we estimate the luminosity and stellar mass functions for different populations of galaxies. Both functions have been extensively investigated in the literature for galaxies of different colors and morphological types (e.g., Lin et al. 1996; Norberg et al. 2002; Madgwick et al. 2002; Blanton et al. 2003b; Bell et al. 2003; Fontana et al. 2006). Note that in a very recent paper, based on the group catalogue constructed from the 2 degree Field Galaxy Redshift Survey (2dFGRS; Colless et al., 2001) by Tago et al. (2006), Tempel et al. (2008) measured luminosity functions for various contents of galaxies (e.g., central, second ranked, satellite, isolated) in groups, as well as for groups. Here we focus on the difference between central and satellite galaxies. We use direct counting to estimate the luminosity function. For each galaxy at a given redshift we calculate its absolute magnitude limit according to Eq. (6). If the absolute magnitude of the galaxy is fainter than this limit, it is removed from the counting list. If the galaxy is not removed, we first calculate the maximum redshift at which the galaxy (with its absolute magnitude) can be observed. We then calculate the comoving volume, $V_{\text {com }}$, between this maximum redshift and a minimum redshift $z=0.01$. In the counting, the galaxy is assigned a weight,

$$
w_{i}=\frac{1}{V_{\mathrm{com}} \mathcal{C}}
$$

where $\mathcal{C}$ is the redshift completeness factor in the NYUVAGC. We calculate the luminosity functions for all, red, and blue galaxies, respectively. The corresponding results are shown in the upper-left panel of Fig. 2] where open circles, squares and triangles are the results for all, red and blue galaxies, respectively. For clarity the results for red and blue galaxies are shifted downwards by a factor of 10. By comparing the LFs for red and blue galaxies, one sees that there are more (fewer) red galaxies than blue ones at $\operatorname{luminosities} \log L \gtrsim 9.8(\log L \lesssim 9.8)$. For very faint galaxies with $\log L \lesssim 9.0$, the red population increase dramatically, exceeding that of blue galaxies at $\log L \sim 8.0$.

We can further separate the galaxies into centrals and satellites according to their memberships in the groups. The corresponding luminosity functions for all, red and blue galaxies are shown in the middle-left and lower-left panels of Fig. 2 2 respectively. The color dependence of the luminosity function for the centrals resembles that of the overall population. However, for the satellite population, the color dependence is somewhat different, especially around $L=10^{9} h^{-2} \mathrm{~L}_{\odot}$, where the luminosity function is not suppressed relative to that of blue satellites, as is the case for the centrals. One interesting feature in the luminosity function of red central galaxies is that there are many very faint red central galaxies (slightly more than the blue ones) with luminosity $\log L \sim 8.0$. Such a population is not expected in the standard galaxy formation models, where very small halos are expected to host only blue centrals. However, Wang, Mo \& Jing (2007) found that the large-scale tidal field may effectively truncate the mass accretion into small halos. If gas accretion is also truncated in this process, the central galaxies in these small halos are expected to be red. More recently, Ludlow et al. (2008) found that some sub-halos that have at some time been within the virial radius of their main progenitors can be ejected, so that some low-mass halos at $z=0$ outside/near the larger virialized halos may have experienced tidal and ram-pressure stripping, so that they have stopped forming stars. In both scenarios, a population of faint red galaxies is expected to be present in the vicinity of high density regions but outside large, virialized halos. This population may be responsible for the upturn of the luminosity function of red central galaxies at the faint end. In a separate paper (Wang et al. 2008b), we will discuss the properties and spatial clustering of the faint red population in more detail, and check the contamination due to false groups near massive ones using mock galaxy and group catalogs.

We also measure the stellar mass functions separately for all, red, blue, central and satellite galaxies, taking into account the stellar mass completeness limit described by Eq.(7). Only galaxies with stellar masses above the completeness limit are used in the estimate. In the right panels of Fig. 2, we show the stellar mass functions using the same symbols as in the left panels. The general behaviors of the stellar mass functions are very similar to the corresponding luminosity functions. For reference, we list the luminosity functions in Table 1, and the stellar mass functions in Table 2,

We use the following Schechter function to fit the lu- 
TABLE 1

The galaXy Luminosity FunCtions $\Phi(L)$

\begin{tabular}{|c|c|c|c|c|c|c|c|c|c|}
\hline \multirow[b]{2}{*}{$\begin{array}{c}(1) \\
\log L\end{array}$} & \multicolumn{3}{|c|}{ ALL } & \multicolumn{3}{|c|}{ CENTRAL } & \multicolumn{3}{|c|}{ SATELLITE } \\
\hline & $\begin{array}{l}\text { (2) } \\
\text { all }\end{array}$ & $\begin{array}{l}(3) \\
\text { red }\end{array}$ & $\begin{array}{c}(4) \\
\text { blue }\end{array}$ & $\begin{array}{l}(5) \\
\text { all }\end{array}$ & $\begin{array}{l}(6) \\
\text { red }\end{array}$ & $\begin{array}{c}(7) \\
\text { blue }\end{array}$ & $\begin{array}{l}\text { (8) } \\
\text { all }\end{array}$ & $\begin{array}{l}(9) \\
\text { red }\end{array}$ & $\begin{array}{l}(10) \\
\text { blue }\end{array}$ \\
\hline 7.8 & $9.0324 \pm 3.3897$ & $5.3944 \pm 2.6814$ & $3.6379 \pm 2.0358$ & $7.0216 \pm 3.2849$ & $4.1403 \pm 2.6068$ & $2.8813 \pm 1.9104$ & $2.0108 \pm 1.6054$ & $1.2542 \pm 0.9849$ & $0.7566 \pm 1.0084$ \\
\hline 7.9 & $7.5462 \pm 1.3850$ & $4.5484 \pm 1.1735$ & $2.9977 \pm 0.7789$ & $4.8617 \pm 1.5245$ & $2.4132 \pm 1.0153$ & $2.4485 \pm 0.8946$ & $2.6845 \pm 1.5813$ & $2.1353 \pm 1.3264$ & $0.5492 \pm 0.5109$ \\
\hline 8.0 & $6.3651 \pm 0.8115$ & $2.8845 \pm 0.5102$ & $3.4806 \pm 0.6290$ & $4.5706 \pm 0.8858$ & $2.0541 \pm 0.5558$ & $2.5166 \pm 0.6278$ & $1.7945 \pm 0.8846$ & $0.8305 \pm 0.5089$ & $0.9640 \pm 0.5210$ \\
\hline 8.1 & $6.0813 \pm 0.6498$ & $2.5566 \pm 0.3424$ & $3.5247 \pm 0.5074$ & $4.4285 \pm 0.7806$ & $1.7563 \pm 0.3504$ & $2.6723 \pm 0.5992$ & $1.6528 \pm 0.7226$ & $0.8003 \pm 0.2809$ & $0.8524 \pm 0.5526$ \\
\hline 8.2 & $4.2781 \pm 0.4229$ & $1.8413 \pm 0.2858$ & $2.4368 \pm 0.2576$ & $2.7779 \pm 0.5448$ & $1.0132 \pm 0.2620$ & $1.7647 \pm 0.3909$ & $1.5002 \pm 0.5502$ & $0.8281 \pm 0.3306$ & $0.6721 \pm 0.3188$ \\
\hline 8.3 & $4.9823 \pm 0.4415$ & $1.8511 \pm 0.2813$ & $3.1312 \pm 0.2737$ & $3.4498 \pm 0.5347$ & $1.0388 \pm 0.1995$ & $2.4110 \pm 0.4263$ & $1.5326 \pm 0.5281$ & $0.8124 \pm 0.2851$ & $0.7202 \pm 0.3201$ \\
\hline 8.4 & $4.5373 \pm 0.3176$ & $1.4754 \pm 0.1732$ & $3.0620 \pm 0.2409$ & $3.0877 \pm 0.4414$ & $0.7873 \pm 0.1432$ & $2.3004 \pm 0.3883$ & $1.4496 \pm 0.4447$ & $0.6881 \pm 0.1755$ & $0.7615 \pm 0.3339$ \\
\hline 8.5 & $4.7646 \pm 0.2712$ & $1.4727 \pm 0.1820$ & $3.2919 \pm 0.1862$ & $3.2717 \pm 0.3598$ & $0.8272 \pm 0.0833$ & $2.4445 \pm 0.3416$ & $1.4930 \pm 0.3578$ & $0.6455 \pm 0.1481$ & $0.8474 \pm 0.2594$ \\
\hline 8.6 & $5.0611 \pm 0.1803$ & $1.3903 \pm 0.1616$ & $3.6708 \pm 0.2012$ & $3.4916 \pm 0.3415$ & $0.6852 \pm 0.0744$ & $2.8064 \pm 0.3192$ & $1.5695 \pm 0.3034$ & $0.7051 \pm 0.1422$ & $0.8644 \pm 0.2057$ \\
\hline 8.7 & $4.9694 \pm 0.1665$ & $1.5349 \pm 0.1161$ & $3.4344 \pm 0.1741$ & $3.1904 \pm 0.3180$ & $0.6093 \pm 0.0819$ & $2.5811 \pm 0.2763$ & $1.7790 \pm 0.2697$ & $0.9256 \pm 0.1269$ & $0.8534 \pm 0.1816$ \\
\hline 8.8 & $4.7649 \pm 0.1504$ & $1.3059 \pm 0.1176$ & $3.4589 \pm 0.1882$ & $3.0336 \pm 0.3153$ & $0.5235 \pm 0.0731$ & $2.5102 \pm 0.2791$ & $1.7313 \pm 0.2625$ & $0.7825 \pm 0.1343$ & $0.9488 \pm 0.1568$ \\
\hline 8.9 & $4.2500 \pm 0.1426$ & $1.2642 \pm 0.0785$ & $2.9857 \pm 0.1575$ & $2.7792 \pm 0.2663$ & $0.5656 \pm 0.0578$ & $2.2136 \pm 0.2340$ & $1.4708 \pm 0.2005$ & $0.6987 \pm 0.0876$ & $0.7721 \pm 0.1348$ \\
\hline 9.0 & $3.7151 \pm 0.1449$ & $1.0853 \pm 0.0615$ & $2.6298 \pm 0.1556$ & $2.4177 \pm 0.2373$ & $0.4832 \pm 0.0559$ & $1.9345 \pm 0.1985$ & $1.2974 \pm 0.1458$ & $0.6021 \pm 0.0827$ & $0.6953 \pm 0.0795$ \\
\hline 9.1 & $3.4459 \pm 0.1257$ & $1.0891 \pm 0.0444$ & $2.3568 \pm 0.1293$ & $2.2779 \pm 0.2120$ & $0.5214 \pm 0.0585$ & $1.7565 \pm 0.1743$ & $1.1680 \pm 0.1280$ & $0.5677 \pm 0.0704$ & $0.6003 \pm 0.0713$ \\
\hline 9.2 & $3.2127 \pm 0.1361$ & $1.0609 \pm 0.0291$ & $2.1518 \pm 0.1267$ & $2.1378 \pm 0.1763$ & $0.5271 \pm 0.0433$ & $1.6106 \pm 0.1480$ & $1.0749 \pm 0.0778$ & $0.5337 \pm 0.0439$ & $0.5412 \pm 0.0465$ \\
\hline 9.3 & $2.8792 \pm 0.0998$ & $1.0113 \pm 0.0308$ & $1.8679 \pm 0.0993$ & $1.9424 \pm 0.1626$ & $0.5276 \pm 0.0440$ & $1.4148 \pm 0.1316$ & $0.9367 \pm 0.0924$ & $0.4836 \pm 0.0505$ & $0.4531 \pm 0.0507$ \\
\hline 9.4 & $2.7471 \pm 0.0998$ & $1.0501 \pm 0.0278$ & $1.6970 \pm 0.0879$ & $1.8702 \pm 0.1530$ & $0.5831 \pm 0.0500$ & $1.2870 \pm 0.1125$ & $0.8770 \pm 0.0756$ & $0.4670 \pm 0.0434$ & $0.4100 \pm 0.0393$ \\
\hline 9.5 & $2.6091 \pm 0.0972$ & $1.0723 \pm 0.0296$ & $1.5368 \pm 0.0783$ & $1.8150 \pm 0.1525$ & $0.6253 \pm 0.0520$ & $1.1897 \pm 0.1094$ & $0.7941 \pm 0.0729$ & $0.4470 \pm 0.0362$ & $0.3471 \pm 0.0430$ \\
\hline 9.6 & $2.6861 \pm 0.0956$ & $1.2200 \pm 0.0332$ & $1.4660 \pm 0.0716$ & $1.8920 \pm 0.1553$ & $0.7424 \pm 0.0642$ & $1.1496 \pm 0.0988$ & $0.7940 \pm 0.0747$ & $0.4776 \pm 0.0433$ & $0.3164 \pm 0.0368$ \\
\hline 9.7 & $2.4858 \pm 0.0875$ & $1.2074 \pm 0.0339$ & $1.2784 \pm 0.0616$ & $1.7868 \pm 0.1444$ & $0.7688 \pm 0.0662$ & $1.0180 \pm 0.0856$ & $0.6990 \pm 0.0697$ & $0.4386 \pm 0.0419$ & $0.2604 \pm 0.0315$ \\
\hline 9.8 & $2.1931 \pm 0.0757$ & $1.1136 \pm 0.0334$ & $1.0795 \pm 0.0491$ & $1.6238 \pm 0.1239$ & $0.7524 \pm 0.0579$ & $0.8713 \pm 0.0715$ & $0.5693 \pm 0.0564$ & $0.3611 \pm 0.0320$ & $0.2082 \pm 0.0279$ \\
\hline 9.9 & $1.8351 \pm 0.0654$ & $0.9485 \pm 0.0274$ & $0.8866 \pm 0.0428$ & $1.4036 \pm 0.1038$ & $0.6725 \pm 0.0489$ & $0.7311 \pm 0.0593$ & $0.4315 \pm 0.0450$ & $0.2760 \pm 0.0269$ & $0.1555 \pm 0.0205$ \\
\hline 10.0 & $1.5384 \pm 0.0557$ & $0.8246 \pm 0.0255$ & $0.7138 \pm 0.0340$ & $1.1979 \pm 0.0884$ & $0.6080 \pm 0.0452$ & $0.5899 \pm 0.0465$ & $0.3405 \pm 0.0383$ & $0.2165 \pm 0.0241$ & $0.1239 \pm 0.0160$ \\
\hline 10.1 & $1.2460 \pm 0.0444$ & $0.6707 \pm 0.0210$ & $0.5753 \pm 0.0265$ & $0.9992 \pm 0.0702$ & $0.5149 \pm 0.0364$ & $0.4843 \pm 0.0366$ & $0.2468 \pm 0.0297$ & $0.1558 \pm 0.0186$ & $0.0910 \pm 0.0124$ \\
\hline 10.2 & $0.9413 \pm 0.0320$ & $0.5112 \pm 0.0160$ & $0.4301 \pm 0.0182$ & $0.7774 \pm 0.0525$ & $0.4108 \pm 0.0274$ & $0.3666 \pm 0.0272$ & $0.1639 \pm 0.0229$ & $0.1004 \pm 0.0133$ & $0.0635 \pm 0.0105$ \\
\hline 10.3 & $0.6428 \pm 0.0226$ & $0.3591 \pm 0.0123$ & $0.2837 \pm 0.0120$ & $0.5448 \pm 0.0353$ & $0.3000 \pm 0.0190$ & $0.2448 \pm 0.0177$ & $0.0980 \pm 0.0141$ & $0.0591 \pm 0.0080$ & $0.0389 \pm 0.0067$ \\
\hline 10.4 & $0.3961 \pm 0.0137$ & $0.2302 \pm 0.0084$ & $0.1659 \pm 0.0065$ & $0.3442 \pm 0.0209$ & $0.1995 \pm 0.0121$ & $0.1447 \pm 0.0098$ & $0.0520 \pm 0.0081$ & $0.0307 \pm 0.0046$ & $0.0212 \pm 0.0039$ \\
\hline 10.5 & $0.2227 \pm 0.0075$ & $0.1393 \pm 0.0053$ & $0.0834 \pm 0.0030$ & $0.1981 \pm 0.0104$ & $0.1241 \pm 0.0067$ & $0.0740 \pm 0.0044$ & $0.0246 \pm 0.0035$ & $0.0153 \pm 0.0020$ & $0.0094 \pm 0.0018$ \\
\hline 10.6 & $0.1078 \pm 0.0028$ & $0.0733 \pm 0.0030$ & $0.0345 \pm 0.0010$ & $0.0987 \pm 0.0042$ & $0.0674 \pm 0.0036$ & $0.0313 \pm 0.0011$ & $0.0091 \pm 0.0018$ & $0.0059 \pm 0.0009$ & $0.0032 \pm 0.0011$ \\
\hline 10.7 & $0.0475 \pm 0.0010$ & $0.0345 \pm 0.0012$ & $0.0130 \pm 0.0011$ & $0.0447 \pm 0.0012$ & $0.0328 \pm 0.0013$ & $0.0119 \pm 0.0006$ & $0.0027 \pm 0.0008$ & $0.0017 \pm 0.0003$ & $0.0011 \pm 0.0006$ \\
\hline 10.8 & $0.0183 \pm 0.0011$ & $0.0140 \pm 0.0006$ & $0.0043 \pm 0.0010$ & $0.0175 \pm 0.0010$ & $0.0135 \pm 0.0006$ & $0.0040 \pm 0.0009$ & $0.0008 \pm 0.0003$ & $0.0005 \pm 0.0001$ & $0.0003 \pm 0.0002$ \\
\hline 10.9 & $0.0060 \pm 0.0006$ & $0.0047 \pm 0.0003$ & $0.0013 \pm 0.0004$ & $0.0059 \pm 0.0005$ & $0.0046 \pm 0.0003$ & $0.0013 \pm 0.00$ & $0.0001 \pm 0.0001$ & $0.0000 \pm 0.0000$ & $0.0000 \pm 0.0001$ \\
\hline 11.0 & $0.0013 \pm 0.0002$ & $0.0011 \pm 0.0002$ & $0.0002 \pm 0.0001$ & $0.0013 \pm 0.0002$ & $0.0011 \pm 0.0002$ & $0.0002 \pm 0.0001$ & $0.0000 \pm 0.0000$ & $0.0000 \pm 0.0000$ & $0.0000 \pm 0.0000$ \\
\hline
\end{tabular}

group members. Columns $(5-7)$ : the luminosity functions for all, red and blue galaxies for 'CENTRAL' group members. Columns ( $8-10)$ : the luminosity functions of all, red and blue galaxies for 'SATELLITE' group members, respectively. Note that all the galaxy luminosity functions listed in this table are in units of $10^{-2} h^{3} \mathrm{Mpc}{ }^{-3} \mathrm{~d} \log L$, where $\log$ is the $10 \mathrm{based} \operatorname{logarithm}$. 
TABLE 2

The Galaxy Stellar Mass Functions $\Phi\left(M_{*}\right)$

\begin{tabular}{|c|c|c|c|c|c|c|c|c|c|}
\hline \multirow[b]{2}{*}{$\begin{array}{c}(1) \\
\log M_{*}\end{array}$} & \multicolumn{3}{|c|}{ 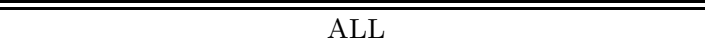 } & \multicolumn{3}{|c|}{ בENTRAL } & \multicolumn{3}{|c|}{ SATELLITE } \\
\hline & $\begin{array}{l}(2) \\
\text { all }\end{array}$ & $\begin{array}{l}(3) \\
\text { red }\end{array}$ & $\begin{array}{c}(4) \\
\text { blue }\end{array}$ & $\begin{array}{l}(5) \\
\text { all }\end{array}$ & $\begin{array}{l}(6) \\
\text { red }\end{array}$ & $\begin{array}{c}(7) \\
\text { blue }\end{array}$ & $\begin{array}{l}(8) \\
\text { all }\end{array}$ & $\begin{array}{l}(9) \\
\text { red }\end{array}$ & $\begin{array}{l}(10) \\
\text { blue }\end{array}$ \\
\hline 8.2 & $6.7578 \pm 3.5635$ & $5.0225 \pm 3.4088$ & $1.7354 \pm 1.3697$ & $5.4097 \pm 3.6339$ & $3.6743 \pm 3.1606$ & $1.7354 \pm 1.3697$ & $1.3481 \pm 1.4459$ & $1.3481 \pm 1.4459$ & $0.0000 \pm 0.0000$ \\
\hline 8.3 & $5.0065 \pm 1.1628$ & $2.6774 \pm 0.9420$ & $2.3291 \pm 0.8917$ & $2.4939 \pm 0.7368$ & $1.3292 \pm 0.6210$ & $1.1647 \pm 0.6085$ & $2.5126 \pm 0.7970$ & $1.3483 \pm 0.5956$ & $1.1644 \pm 0.5844$ \\
\hline 8.4 & $5.2026 \pm 0.8331$ & $1.8689 \pm 0.5549$ & $3.3337 \pm 0.5824$ & $3.7970 \pm 0.8574$ & $0.9167 \pm 0.3865$ & $2.8803 \pm 0.6688$ & $1.4056 \pm 0.7744$ & $0.9522 \pm 0.5715$ & $0.4534 \pm 0.3364$ \\
\hline 8.5 & $3.6943 \pm 0.6310$ & $1.3602 \pm 0.3937$ & $2.3340 \pm 0.4160$ & $2.8124 \pm 0.5254$ & $0.8612 \pm 0.2399$ & $1.9512 \pm 0.4411$ & $0.8819 \pm 0.5271$ & $0.4990 \pm 0.3365$ & $0.3829 \pm 0.2879$ \\
\hline 8.6 & $3.2254 \pm 0.4123$ & $0.9867 \pm 0.2701$ & $2.2387 \pm 0.3139$ & $2.4063 \pm 0.4036$ & $0.5364 \pm 0.1443$ & $1.8699 \pm 0.3612$ & $0.8191 \pm 0.3452$ & $0.4503 \pm 0.2265$ & $0.3687 \pm 0.1858$ \\
\hline 8.7 & $3.1947 \pm 0.3411$ & $0.8821 \pm 0.2339$ & $2.3126 \pm 0.2237$ & $2.0724 \pm 0.3095$ & $0.4822 \pm 0.1251$ & $1.5902 \pm 0.2715$ & $1.1223 \pm 0.3426$ & $0.3999 \pm 0.1989$ & $0.7224 \pm 0.2100$ \\
\hline 8.8 & $2.7979 \pm 0.2362$ & $0.8262 \pm 0.1388$ & $1.9717 \pm 0.2255$ & $2.0071 \pm 0.2667$ & $0.3576 \pm 0.0824$ & $1.6495 \pm 0.2453$ & $0.7908 \pm 0.1654$ & $0.4687 \pm 0.1130$ & $0.3222 \pm 0.0997$ \\
\hline 8.9 & $2.7209 \pm 0.1921$ & $0.9936 \pm 0.1432$ & $1.7273 \pm 0.1742$ & $1.8136 \pm 0.2374$ & $0.4987 \pm 0.0766$ & $1.3149 \pm 0.2136$ & $0.9073 \pm 0.1880$ & $0.4949 \pm 0.1142$ & $0.4124 \pm 0.1215$ \\
\hline 9.0 & $2.5366 \pm 0.1544$ & $0.7511 \pm 0.1110$ & $1.7855 \pm 0.1573$ & $1.7138 \pm 0.1638$ & $0.3434 \pm 0.0637$ & $1.3704 \pm 0.1687$ & $0.8228 \pm 0.1219$ & $0.4076 \pm 0.0788$ & $0.4151 \pm 0.0770$ \\
\hline 9.1 & $2.8001 \pm 0.1436$ & $0.9423 \pm 0.1323$ & $1.8578 \pm 0.1762$ & $1.8134 \pm 0.2080$ & $0.3792 \pm 0.0744$ & $1.4342 \pm 0.1698$ & $0.9867 \pm 0.1670$ & $0.5630 \pm 0.1391$ & $0.4236 \pm 0.0625$ \\
\hline 9.2 & $2.5299 \pm 0.1329$ & $0.8579 \pm 0.1015$ & $1.6720 \pm 0.1546$ & $1.6551 \pm 0.1690$ & $0.3732 \pm 0.0545$ & $1.2819 \pm 0.1469$ & $0.8748 \pm 0.1109$ & $0.4847 \pm 0.0974$ & $0.3901 \pm 0.0431$ \\
\hline 9.3 & $2.6046 \pm 0.1105$ & $0.8839 \pm 0.0824$ & $1.7207 \pm 0.1232$ & $1.7112 \pm 0.1675$ & $0.4203 \pm 0.0540$ & $1.2908 \pm 0.1388$ & $0.8935 \pm 0.1283$ & $0.4636 \pm 0.0881$ & $0.4299 \pm 0.0612$ \\
\hline 9.4 & $2.6018 \pm 0.1118$ & $1.0060 \pm 0.0732$ & $1.5958 \pm 0.1207$ & $1.6445 \pm 0.1602$ & $0.4455 \pm 0.0551$ & $1.1990 \pm 0.1283$ & $0.9573 \pm 0.1105$ & $0.5605 \pm 0.0818$ & $0.3968 \pm 0.0480$ \\
\hline 9.5 & $2.2135 \pm 0.1075$ & $0.8682 \pm 0.0492$ & $1.3453 \pm 0.1048$ & $1.4016 \pm 0.1165$ & $0.4033 \pm 0.0414$ & $0.9983 \pm 0.0926$ & $0.8118 \pm 0.0559$ & $0.4649 \pm 0.0533$ & $0.3470 \pm 0.0317$ \\
\hline 9.6 & $2.1244 \pm 0.0969$ & $0.9046 \pm 0.0347$ & $1.2197 \pm 0.0840$ & $1.4653 \pm 0.1207$ & $0.4732 \pm 0.0443$ & $0.9921 \pm 0.0951$ & $0.6590 \pm 0.0572$ & $0.4314 \pm 0.0407$ & $0.2276 \pm 0.0288$ \\
\hline 9.7 & $1.9695 \pm 0.0934$ & $0.9288 \pm 0.0355$ & $1.0407 \pm 0.0762$ & $1.3411 \pm 0.1129$ & $0.5037 \pm 0.0502$ & $0.8374 \pm 0.0763$ & $0.6284 \pm 0.0531$ & $0.4251 \pm 0.0434$ & $0.2033 \pm 0.0194$ \\
\hline 9.8 & $1.7968 \pm 0.0687$ & $0.8633 \pm 0.0252$ & $0.9335 \pm 0.0614$ & $1.2160 \pm 0.0882$ & $0.4676 \pm 0.0395$ & $0.7483 \pm 0.0616$ & $0.5808 \pm 0.0451$ & $0.3956 \pm 0.0395$ & $0.1852 \pm 0.0144$ \\
\hline 9.9 & $1.7543 \pm 0.0808$ & $0.9275 \pm 0.0410$ & $0.8268 \pm 0.0538$ & $1.1954 \pm 0.0921$ & $0.5415 \pm 0.0491$ & $0.6539 \pm 0.0545$ & $0.5589 \pm 0.0340$ & $0.3859 \pm 0.0271$ & $0.1729 \pm 0.0134$ \\
\hline 10.0 & $1.6529 \pm 0.0658$ & $0.9104 \pm 0.0335$ & $0.7426 \pm 0.0427$ & $1.1378 \pm 0.0845$ & $0.5414 \pm 0.0472$ & $0.5963 \pm 0.0451$ & $0.5152 \pm 0.0338$ & $0.3689 \pm 0.0283$ & $0.1462 \pm 0.0115$ \\
\hline 10.1 & $1.6162 \pm 0.0660$ & $0.9446 \pm 0.0356$ & $0.6716 \pm 0.0398$ & $1.1427 \pm 0.0837$ & $0.5939 \pm 0.0483$ & $0.5488 \pm 0.0431$ & $0.4735 \pm 0.0316$ & $0.3507 \pm 0.0264$ & $0.1228 \pm 0.0100$ \\
\hline 10.2 & $1.5700 \pm 0.0631$ & $0.9791 \pm 0.0388$ & $0.5908 \pm 0.0340$ & $1.1120 \pm 0.0814$ & $0.6402 \pm 0.0531$ & $0.4718 \pm 0.0351$ & $0.4580 \pm 0.0310$ & $0.3389 \pm 0.0276$ & $0.1190 \pm 0.0076$ \\
\hline 10.3 & $1.5025 \pm 0.0564$ & $0.9766 \pm 0.0325$ & $0.5259 \pm 0.0298$ & $1.0918 \pm 0.0766$ & $0.6635 \pm 0.0503$ & $0.4284 \pm 0.0320$ & $0.4107 \pm 0.0308$ & $0.3132 \pm 0.0271$ & $0.0975 \pm 0.0066$ \\
\hline 10.4 & $1.3108 \pm 0.0480$ & $0.8909 \pm 0.0302$ & $0.4199 \pm 0.0230$ & $0.9663 \pm 0.0682$ & $0.6177 \pm 0.0479$ & $0.3486 \pm 0.0252$ & $0.3445 \pm 0.0285$ & $0.2732 \pm 0.0247$ & $0.0713 \pm 0.0057$ \\
\hline 10.5 & $1.0826 \pm 0.0356$ & $0.7608 \pm 0.0245$ & $0.3218 \pm 0.0155$ & $0.8213 \pm 0.0529$ & $0.5522 \pm 0.0384$ & $0.2691 \pm 0.0186$ & $0.2613 \pm 0.0237$ & $0.2087 \pm 0.0197$ & $0.0527 \pm 0.0057$ \\
\hline 10.6 & $0.8499 \pm 0.0267$ & $0.6080 \pm 0.0188$ & $0.2419 \pm 0.0111$ & $0.6604 \pm 0.0418$ & $0.4549 \pm 0.0309$ & $0.2055 \pm 0.0138$ & $0.1896 \pm 0.0193$ & $0.1532 \pm 0.0159$ & $0.0364 \pm 0.0046$ \\
\hline 10.7 & $0.6440 \pm 0.0193$ & $0.4772 \pm 0.0145$ & $0.1667 \pm 0.0074$ & $0.5182 \pm 0.0311$ & $0.3753 \pm 0.0242$ & $0.1429 \pm 0.0092$ & $0.1257 \pm 0.0151$ & $0.1019 \pm 0.0129$ & $0.0238 \pm 0.0032$ \\
\hline 10.8 & $0.4582 \pm 0.0130$ & $0.3556 \pm 0.0107$ & $0.1026 \pm 0.0039$ & $0.3811 \pm 0.0211$ & $0.2910 \pm 0.0174$ & $0.0901 \pm 0.0051$ & $0.0771 \pm 0.0101$ & $0.0646 \pm 0.0087$ & $0.0125 \pm 0.0020$ \\
\hline 10.9 & $0.3009 \pm 0.0071$ & $0.2436 \pm 0.0069$ & $0.0573 \pm 0.0014$ & $0.2572 \pm 0.0123$ & $0.2064 \pm 0.0114$ & $0.0508 \pm 0.0019$ & $0.0437 \pm 0.0067$ & $0.0373 \pm 0.0059$ & $0.0064 \pm 0.0012$ \\
\hline 11.0 & $0.1846 \pm 0.0047$ & $0.1573 \pm 0.0048$ & $0.0273 \pm 0.0011$ & $0.1627 \pm 0.0073$ & $0.1379 \pm 0.0071$ & $0.0248 \pm 0.0008$ & $0.0219 \pm 0.0035$ & $0.0194 \pm 0.0031$ & $0.0025 \pm 0.0006$ \\
\hline 11.1 & $0.1066 \pm 0.0022$ & $0.0931 \pm 0.0027$ & $0.0135 \pm 0.0012$ & $0.0964 \pm 0.0037$ & $0.0839 \pm 0.0039$ & $0.0125 \pm 0.0008$ & $0.0102 \pm 0.0020$ & $0.0092 \pm 0.0016$ & $0.0010 \pm 0.0005$ \\
\hline 11.2 & $0.0575 \pm 0.0011$ & $0.0520 \pm 0.0017$ & $0.0055 \pm 0.0009$ & $0.0531 \pm 0.0018$ & $0.0480 \pm 0.0023$ & $0.0052 \pm 0.0007$ & $0.0044 \pm 0.0010$ & $0.0040 \pm 0.0008$ & $0.0003 \pm 0.0002$ \\
\hline 11.3 & $0.0294 \pm 0.0006$ & $0.0271 \pm 0.0009$ & $0.0023 \pm 0.0004$ & $0.0281 \pm 0.0009$ & $0.0258 \pm 0.0011$ & $0.0022 \pm 0.0004$ & $0.0014 \pm 0.0004$ & $0.0013 \pm 0.0003$ & $0.0001 \pm 0.0001$ \\
\hline 11.4 & $0.0131 \pm 0.0004$ & $0.0122 \pm 0.0005$ & $0.0010 \pm 0.0002$ & $0.0127 \pm 0.0005$ & $0.0117 \pm 0.0005$ & $0.0009 \pm 0.0002$ & $0.0004 \pm 0.0001$ & $0.0004 \pm 0.0001$ & $0.0000 \pm 0.0000$ \\
\hline 11.5 & $0.0047 \pm 0.0003$ & $0.0044 \pm 0.0003$ & $0.0003 \pm 0.0001$ & $0.0047 \pm 0.0003$ & $0.0044 \pm 0.0003$ & $0.0003 \pm 0.0001$ & $0.0000 \pm 0.0000$ & $0.0000 \pm 0.0000$ & $0.0000 \pm 0.0000$ \\
\hline 11.6 & $0.0012 \pm 0.0001$ & $0.0011 \pm 0.0001$ & $0.0001 \pm 0.0000$ & $0.0012 \pm 0.0001$ & $0.0011 \pm 0.0001$ & $0.0001 \pm 0.0000$ & $0.0000 \pm 0.0000$ & $0.0000 \pm 0.0000$ & $0.0000 \pm 0.0000$ \\
\hline
\end{tabular}

NotE. - Column (1): the median of the logarithm of the galaxy stellar mass with bin width $\Delta \log M_{*}=0.05$. Column ( $\left.2-4\right)$ : the stellar mass functions of all, red and blue for 'ALL' group
members. Column $(5-7)$ : the stellar mass functions of all, red and blue for 'CENTRAL' group members. Column ( 8 - 10): the stellar mass functions of all, red and blue for 'SATELLITE' group members. Note that all the galaxy stellar mass functions listed in this table are in units of $10^{-2} h^{3} \mathrm{Mpc}^{-3} \mathrm{~d} \log M_{*}$, where $\log$ is the 10 based $\operatorname{logarithm}$. 

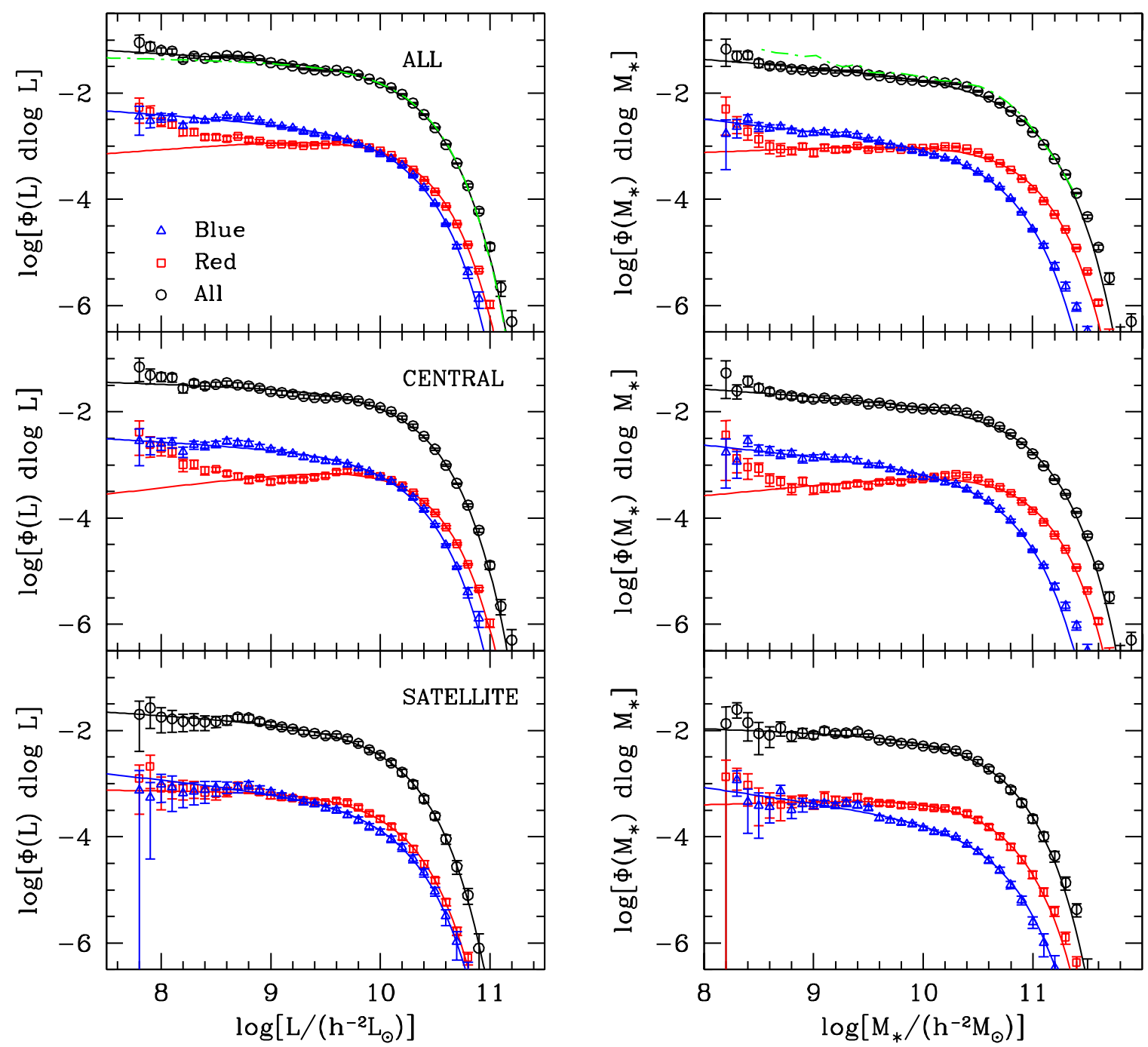

FIG. 2.- The galaxy luminosity functions (left panels) and stellar mass functions (right panels). The upper, middle and lower panels show results obtained from all, central and satellite group members, respectively. In each panel, the open circles, squares and triangles with error-bars are the luminosity or stellar mass functions of all, red and blue galaxies, respectively, where the errors are obtained from the 200 bootstrap samples. Note that the results for red and blue galaxies are scaled down by a factor of 10, for clarity. The solid lines in each panel are the best fitting Schechter functions. For comparison, the dot-dashed line in the upper-left panel is the best fit luminosity function obtained by Blanton et al. (2003b), while that in the upper-right panel corresponds to the stellar mass function obtained by Bell et al. (2003) from the SDSS Early Data Release.

minosity function:

$$
\Phi(L)=\phi^{\star}\left(\frac{L}{L^{\star}}\right)^{(\alpha+1)} \exp \left[-\frac{L}{L^{\star}}\right]
$$

For the stellar mass function luminosity functions, we use a similar model:

$$
\Phi\left(M_{*}\right)=\phi^{\star}\left(\frac{M_{*}}{M^{\star}}\right)^{(\alpha+1)} \exp \left[-\frac{M_{*}}{M^{\star}}\right] .
$$

For each model, there are three free parameters, the amplitude $\phi^{\star}$, the faint end slope $\alpha$ and the characteristic luminosity $L^{\star}$ (or stellar mass $M^{\star}$ ). Using the least $\chi^{2}$ fitting to the measured luminosity and stellar mass functions shown in Fig. 2, we find the best fit values of $\phi^{\star}$, $\alpha, L^{\star}\left(\right.$ or $\left.M^{\star}\right)$, which are listed in Table 3 The best fit results are shown as the solid lines in Fig.2]

Comparing the data with the best fit, one sees that the Schechter form describes the data remarkably well over the luminosity range $\log L \gtrsim 9.0$. At the fainter end, however, the data reveals an upturn, which is almost entirely due to the red centrals. The stellar mass functions also show a steepening at the low mass end, which again owes mainly to the population of red centrals.

The galaxy luminosity and stellar mass functions have been estimated before by various authors. As an illustration, we show the best fit of the luminosity function obtained by Blanton et al. (2003b) from the SDSS DR2 as the dot-dashed line in the upper-left panel of Fig. 2, As expected, our measurements are in excellent agreement with theirs. For the stellar mass function, we show as the dot-dashed line the result of Bell et al. (2003) obtained from the SDSS Early Data Release (EDR; Stoughton et al. 2002). The agreement with our measurement is remarkably good for $\log M_{*} \gtrsim 9.5$. For lower masses, however, the mass function obtained by Bell et al. (2003) is significantly higher. The discrepancy most likely results from the different data samples (DR4 vs. EDR) used in 


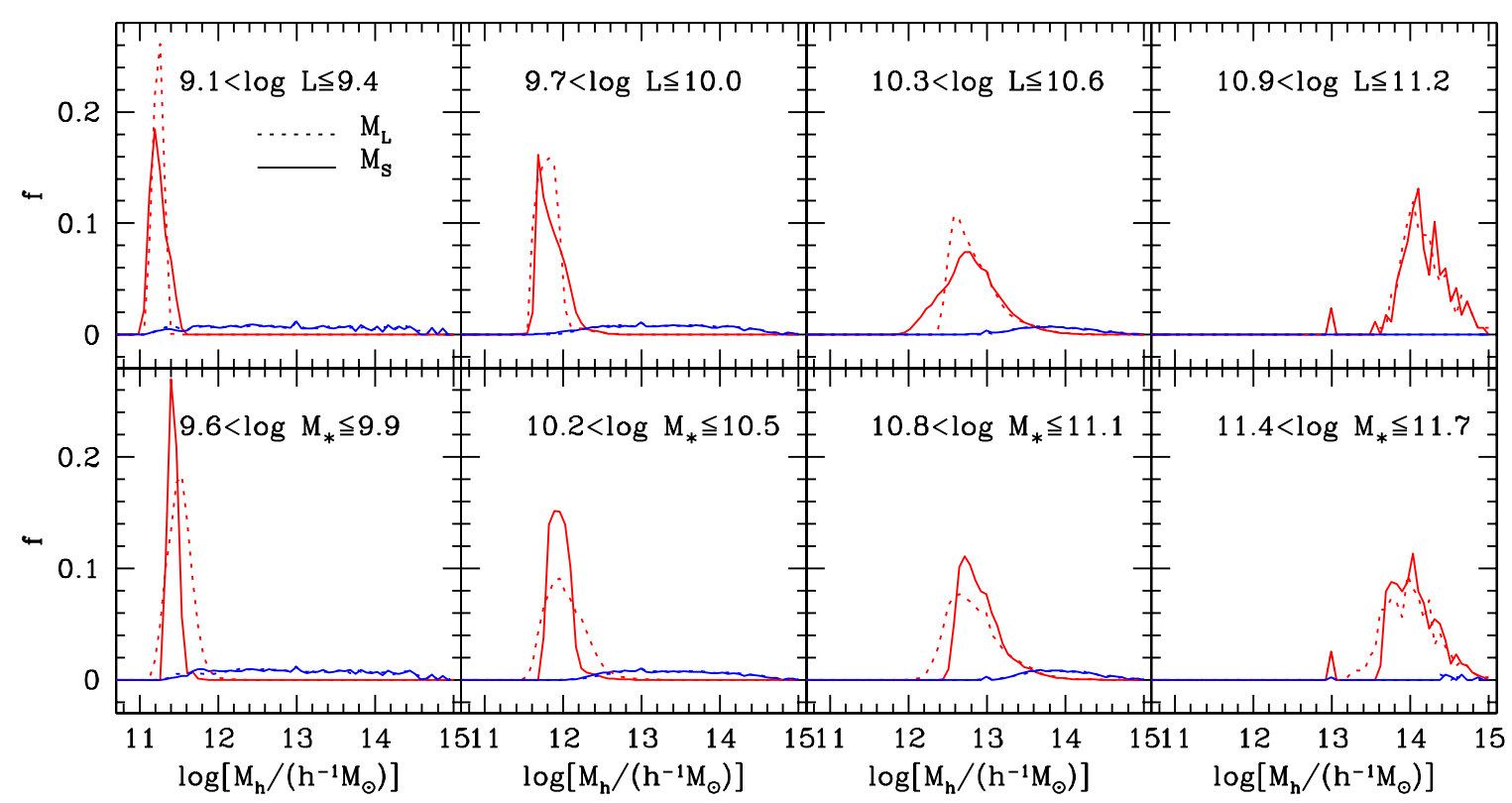

Fig. 3. - The host halo mass distributions for central (red lines) and satellite (blue lines) galaxies. Shown in the upper panels are results for galaxies in different luminosity bins as indicated. Shown in the lower panels are results for galaxies in different stellar mass bins as indicated. The dotted and solid lines represent the results for halo masses, $M_{L}$ and $M_{S}$, respectively.

TABLE 3

THE BEST FIT PARAMETERS FOR THE GALAXY LUMINOSITY FUNCTIONS AND STELLAR MASS FUNCTIONS

\begin{tabular}{llccc}
\hline \hline Member type & color & $\phi^{\star}$ & $\alpha$ & $\log L^{\star}$ \\
\hline$(1)$ & $(2)$ & $(3)$ & $(4)$ & $(5)$ \\
\hline ALL & all & 0.03167 & -1.117 & 10.095 \\
- & red & 0.01810 & -0.846 & 10.080 \\
- & blue & 0.01890 & -1.154 & 10.026 \\
CENTRAL & all & 0.02370 & -1.069 & 10.114 \\
- & red & 0.01223 & -0.755 & 10.106 \\
- & blue & 0.01523 & -1.123 & 10.033 \\
SATELLITE & all & 0.01051 & -1.134 & 9.947 \\
- & red & 0.00682 & -1.018 & 9.923 \\
- & blue & 0.00410 & -1.235 & 9.951 \\
\hline \multirow{2}{*}{ Member type } & color & $\phi^{\star}$ & & \\
\hline \multirow{2}{*}{ ALL } & all & 0.01546 & -1.164 & 10.717 \\
- & red & 0.01285 & -0.916 & 10.691 \\
- & blue & 0.00836 & -1.233 & 10.508 \\
CENTRAL & all & 0.01084 & -1.143 & 10.758 \\
- & red & 0.00903 & -0.803 & 10.717 \\
- & blue & 0.00668 & -1.218 & 10.522 \\
SATELLITE & all & 0.00692 & -1.078 & 10.483 \\
- & red & 0.00589 & -0.932 & 10.457 \\
- & blue & 0.00165 & -1.290 & 10.450 \\
\hline
\end{tabular}

Note. - Column (1): the member type. Column (2): the color of galaxies. Column (3-5): the best fit parameters for the luminosity functions (upper part) and stellar mass functions (lower part). Note that $\phi^{\star}$ listed in column 3 are presented in terms of $h^{3} \mathrm{Mpc}^{-3} \mathrm{~d} \log L$ (or $h^{3} \mathrm{Mpc}^{-3} \mathrm{~d} \log M_{*}$ ), where log is the 10 based logarithm.

the two analyses. For low-mass galaxies, the cosmic variance is significant, especially in EDR because only a few hundred galaxies in a small volume were used to measure the stellar mass function. In addition, we have taken into account the redshift completeness of galaxies using the completeness masks provided by the NYU team, and the stellar mass limit is treated more carefully in our analysis using Eq. 7. The behavior of the stellar mass function in the low-mass end has also been investigated by Baldry et al. (2004, 2008) and Panter et al. (2007). Unfortunately, the situation is still unclear, partly because of the limited sample volume, and partly because of the uncertainties in the luminosity-mass conversion.

To see where galaxies of different luminosities and stellar masses are hosted, we plot in Fig. 3 the host halo mass distribution for central (red lines) and satellite (blue lines) galaxies. Results are shown for galaxies in different luminosity (upper panels) and stellar mass (lower panels) bins, as indicated ${ }^{9}$. Almost all bright (or massive) galaxies are centrals in massive halos. About $3 / 4$ of faint (low-mass) galaxies are centrals in small halos with a very narrow mass distribution, and the rest $1 / 4$ are satellites in halos that cover a wide range in mass. The results are shown separately for halo masses, $M_{L}$ and $M_{S}$. Although the results for satellite galaxies and the mean halo mass for central galaxies are similar for the two halo mass estimates, the widths of the halo mass distribution for central galaxies are quite different. This is caused by the fact that these is some spurious correlation between $L_{19.5}\left(M_{\text {stellar}}\right)$ and the luminosity (stellar mass) of the central galaxy, especially for low-mass halos where the luminosity (stellar) content is dominated by the central galaxy. We have measured the halo mass distribution from both samples II and III, and we do not find any significant difference between the results. Therefore only the results for sample II are plotted in Fig 3 . The general behavior of the halo mass distribution of the central and satellite galaxies is consistent with that predicted by the CLF and HOD models (e.g. Yang et al.

\footnotetext{
9 Note that in the group catalog, halo masses are provided only for groups whose central galaxies are brighter than ${ }^{0.1} \mathrm{M}_{r}-5 \log h=$ -19.5. For groups with a fainter central galaxy we use the mean mass-to-light (Eq19) and halo-to-stellar mass (Eq20) ratios obtained in Section [6] to estimate their halo masses.
} 
2003; Zheng et al. 2005).

\section{THE CONDITIONAL STELLAR MASS FUNCTION}

In paper II, we have measured the conditional luminosity function (CLF) of galaxies in halos (as represented by galaxy groups), $\Phi\left(L \mid M_{h}\right)$. Here we first obtain the conditional stellar mass function (CSMF) of galaxies in dark halos. The CSMF, $\Phi\left(M_{*} \mid M_{h}\right)$, which describes the average number of galaxies as a function of galaxy stellar mass in a dark matter halo of a given mass, is more straightforwardly related to theoretical predictions of galaxy formation models than the CLF, because the conversion from stellar mass to luminosity in theoretical models requires detailed modeling of the stellar population and dust extinction. The CSMF can be estimated by directly counting the number of galaxies in groups. However, because of the completeness limits discussed in Section 2.2, we only use galaxies and groups that are complete according to Eqs. (7) and (9) to estimate the CSMF, $\Phi\left(M_{*} \mid M_{h}\right)$, at a given $M_{*}$. In Fig. 4 we show the resulting CSMFs for groups of different masses. The contributions of central and satellite galaxies are plotted separately. For comparison, results obtained using $M_{S}$ and $M_{L}$ are shown as symbols and dashed lines, respectively. The error-bars shown in each panel correspond to $1-\sigma$ scatter obtained from 200 bootstrap samples of our group catalogue. In general, these two halo masses give consistent results, except that the $M_{S}$-based CSMF of the central galaxies in low mass halos is more peaked than the $M_{L}$-based CSMF (see the lower right-hand panel). The general behavior of the CSMF is similar to that of the CLF presented in Paper II. The general behavior of the CSMF obtained here is also qualitatively similar to the prediction of semi-analytical models (e.g. Zheng et al. 2005): the CSMF for small halos has a strong peak at the bright end due to central galaxies. Quantitatively, however, semi-analytical models in general over-predict the number of satellite galaxies (Liu et al. in preparation).

In Fig. 5 we show the CSMFs separately for red (dashed lines with errorbars) and blue (dotted lines) galaxies. Clearly there are more red galaxies than blue galaxies (both centrals and satellites) in massive halos. In the lowest mass bin probed here $\left(12.0<\log M_{h} \leq\right.$ $12.3)$, however, there are roughly equal numbers of red and blue galaxies. The fraction of red galaxies as a function of halo mass found here is very similar to that obtained by Zandivarez et al. (2006) based on the conditional luminosity function of galaxies derived from an independent group catalog. Note that the overall shapes of the CSMFs for red and blue galaxies are remarkably similar. Interestingly, such behavior is predicted by Skibba et al. (2008) who used the color-marked correlation function to constrain the distribution of galaxies according to their colors.

We model the CSMF using the sum of the CSMFs of central and satellite galaxies (see Yang et al. 2003; Cooray 2005; White et al. 2007; Zheng et al. 2007; Yang et al. 2008; Cacciato et al. 2008):

$$
\Phi\left(M_{*} \mid M_{h}\right)=\Phi_{\text {cen }}\left(M_{*} \mid M_{h}\right)+\Phi_{\text {sat }}\left(M_{*} \mid M_{h}\right) .
$$

Following Paper II, we adopt a lognormal model for the
CSMF of central galaxies:

$$
\Phi_{\text {cen }}\left(M_{*} \mid M_{h}\right)=\frac{A}{\sqrt{2 \pi} \sigma_{c}} \exp \left[-\frac{\left(\log M_{*}-\log M_{*, c}\right)^{2}}{2 \sigma_{c}^{2}}\right],
$$

where $A$ is the number of central galaxies per halo. Thus, $A \equiv 1$ for all galaxies, $A=f_{\text {red }}\left(M_{h}\right)$ for red galaxies, and $A=1-f_{\text {red }}\left(M_{h}\right)$ for blue galaxies. Here $f_{\text {red }}\left(M_{h}\right)$ is the red fraction of central galaxies in halos of mass $M_{h}$. Note that $\log M_{*, c}$ is, by definition, the expectation value for the (10-based) logarithm of the stellar mass of the central galaxy:

$$
\log M_{*, c}=\int_{0}^{\infty} \Phi_{\text {cen }}\left(M_{*} \mid M_{h}\right) \log M_{*} \mathrm{~d} \log M_{*},
$$

and that $\sigma_{c}=\sigma\left(\log M_{*}\right)$. For the contribution from the satellite galaxies we adopt a modified Schechter function:

$$
\Phi_{\text {sat }}\left(M_{*} \mid M_{h}\right)=\phi_{s}^{*}\left(\frac{M_{*}}{M_{*, s}}\right)^{\left(\alpha_{s}^{*}+1\right)} \exp \left[-\left(\frac{M_{*}}{M_{*, s}}\right)^{2}\right] .
$$

Note that this function decreases faster at the bright end than a Schechter function and gives a better description of the data. The above parameterization has a total of five free parameters: $M_{*, c}, \sigma_{c}, \phi_{s}^{*}, \alpha_{s}^{*}$ and $M_{*, s}$. We find that $\log M_{*, c}=\log M_{*, s}+0.25$ to good approximation, which is what we adopt throughout. Consequently, the number of free parameters is reduced to four. Note, however, Hansen et al. (2007) found that the ratio between the mean stellar mass (luminosity) of the central galaxy and the characteristic stellar mass (luminosity) of the satellite galaxies depends on halo mass especially for massive halos. The difference may arise from the fact that they are using galaxy groups constructed from the SDSS photometric redshift catalogue where the memberships of the groups are not so well constrained.

We fit the above model to all our CSMFs. Results are shown in Fig 6. separately for all (left panels), red (middle panels) and blue (right panels) galaxies. Here comparison is made between samples II and III. Note that Sample II does not include any galaxies missed due to fiber collisions, while Sample III includes all such galaxies by assigning each of them the redshift of its nearest neighbor. Given that we also have two kinds of halo masses, $M_{L}$ and $M_{S}$, there are a total of four different combinations for which we have determined the CSMFs. Each panel of Fig [6 shows the results for all four cases. As an illustration of how well the model fits the data, the solid lines in Figs. 4 and 5] show the corresponding best-fit models.

The upper row of Fig. 6 shows the best fit normalization of the CSMF for satellite galaxies, which describes the average number of satellite galaxies with stellar mass $\sim M_{*, s}$ in a group of a given halo mass. As expected, Sample III gives a higher $\phi_{s}^{*}$, especially for low-mass groups, but only marginally so. Comparing $\phi_{s}^{*}$ for red (upper middle panel) and blue (upper right panel) galaxies, one sees that the fraction of red satellites increases with halo mass. The second row shows the faint end slopes of the CSMFs, $\alpha_{s}^{*}$. In massive halos with $M_{h} \gtrsim 10^{13} h^{-1} \mathrm{M}_{\odot}, \alpha_{s}^{*}$ decreases (i.e., becomes more negative) with increasing halo mass, both for red and blue galaxies. In halos with $M_{h} \lesssim 10^{13} h^{-1} \mathrm{M}_{\odot}$, however, $\alpha_{s}^{*}$ 

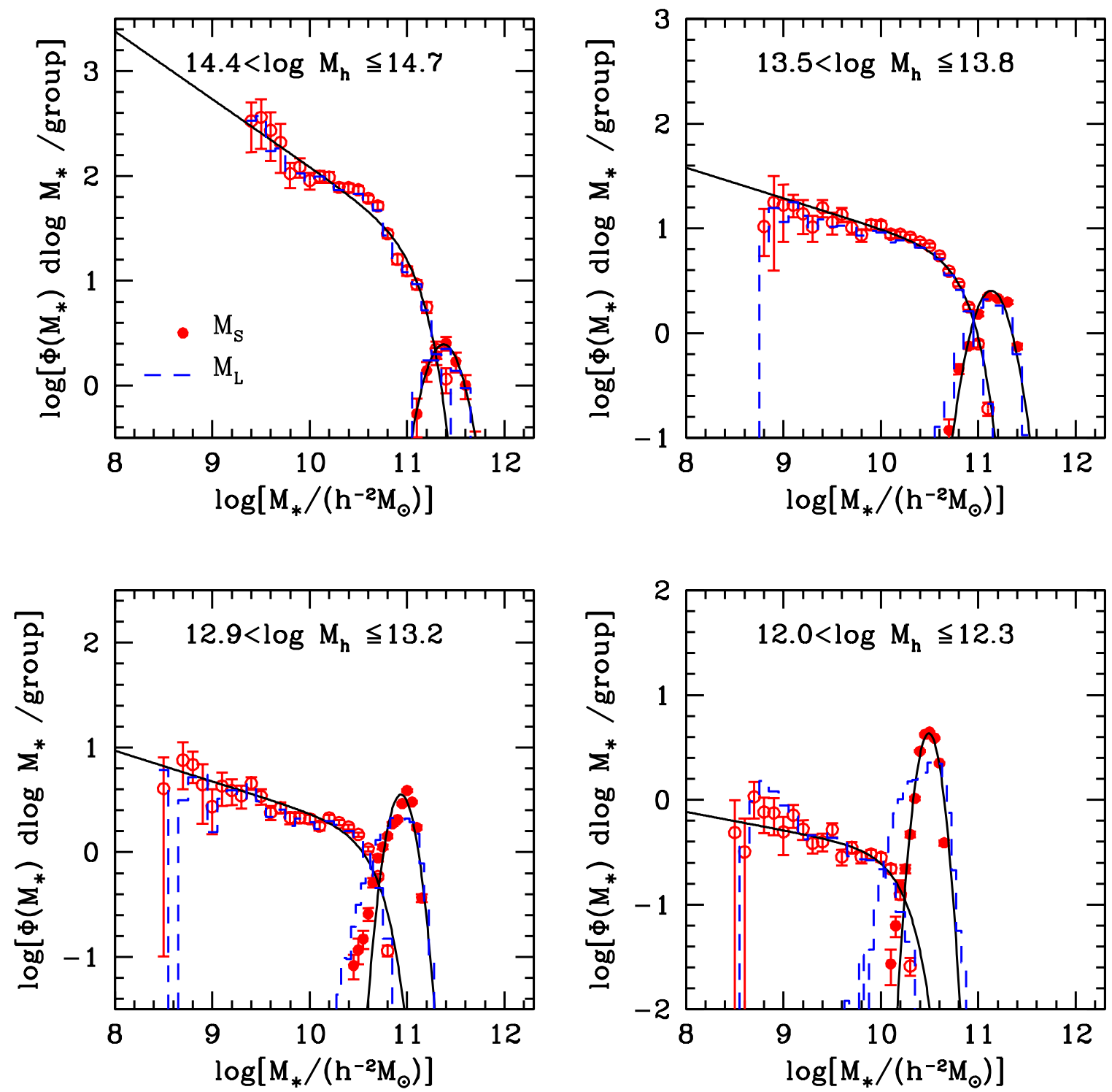

FIG. 4.- The conditional stellar mass functions (CSMFs) of galaxies in groups of different mass bins. Symbols correspond to the CSMFs obtained using $M_{S}$ as halo mass (estimated according to the ranking of the characteristic group stellar masses), with solid and open circles indicating the contributions from central and satellite galaxies, respectively. The error-bars reflect the 1- $\sigma$ scatter obtained from 200 bootstrap samples for $M_{L}$ and $M_{S}$, respectively. The solid lines indicate the best-fit parameterizations (equations 13 to 16 ). For comparison, we also show, with dashed lines, the CSMFs obtained using $M_{L}$ as halo mass (estimated according to the ranking of the characteristic group luminosity).

is roughly constant at $\sim-1.2$. The third row shows that $\log M_{*, c}$ increases with halo mass, for both red and blue centrals. Note that for a given value of $\log M_{*, c}$, the halo mass for blue galaxies based on $M_{L}$ is larger than that based on $M_{S}$, especially in small halos. The reason is that for a given stellar mass, bluer galaxies are brighter, hence $M_{L}$ is higher. This effect can impact (e.g. slightly enhance) our results on the color dependence of group clustering (e.g., Berlind et al. 2006; Wang et al. 2008a). These best fit parameters for satellite galaxies of different colors are different from that obtained from the CLF measurements (e.g. Zandivarez et al. 2006; Hansen et al. 2007; Yang et al. 2008). In particular the low-mass end slope, $\alpha_{s}^{*}$, obtained here for red satellite galaxies in small halos is significantly steeper. The only significant difference between red and blue galaxies is in $\phi_{s}^{*}$, consistent with the results of Skibba (2008). Finally, the last row of Fig 6] shows the width of the log-normal CSMF of central galaxies. For the combined sample of red and blue galaxies we find an average value of $\sigma_{c}=\sigma\left(\log M_{*, c}\right) \sim 0.17$ in halos with $M_{h} \gtrsim 10^{13} h^{-1} \mathrm{M}_{\odot}$. This width is slightly larger than that in the luminosity distribution obtained in Paper II. A roughly constant dispersion in the lognormal distribution of luminosity (or stellar mass) for central galaxies has already been predicted by Yang et al. (2003), Cooray (2006) and Cacciato et al. (2008) from the clustering and abundances of galaxies. Although Zheng et al. (2007) found, based on a HOD model applied to the SDSS and DEEP2, that the log-normal width increases from $\sim 0.13$ for massive halos with $M_{h} \sim 10^{13.5} h^{-1} \mathrm{M}_{\odot}$ to $\sim 0.3$ for low mass halos with $M_{h} \sim 10^{11.5} h^{-1} \mathrm{M}_{\odot}$, the difference between their results and ours is only at 

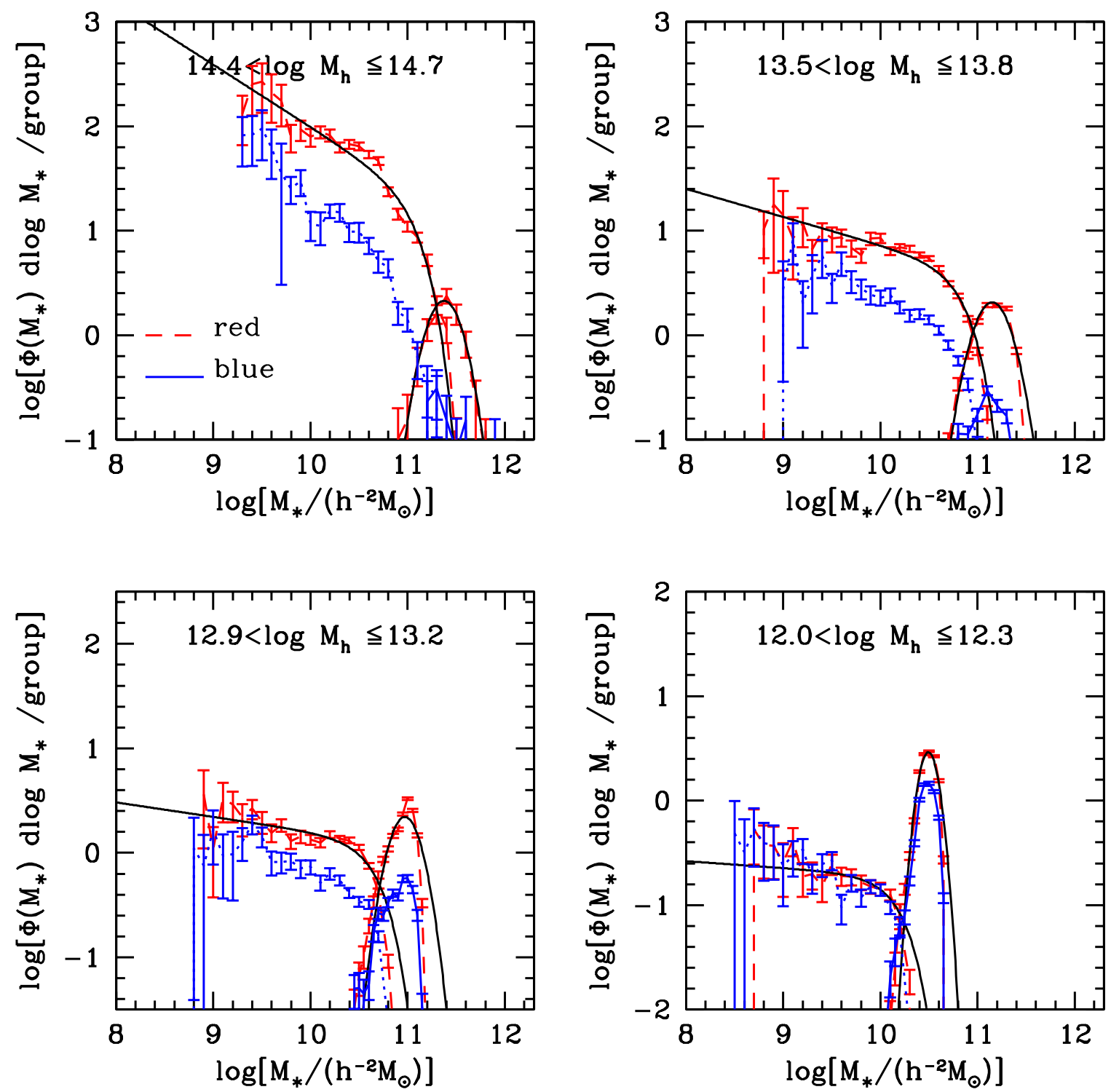

FIG. 5.- Similar to Fig. 4 but here we show the CSMFs for red (dashed lines) and blue (dotted lines) galaxies. In both cases the central and satellite components of the CSMFs are indicated separately. The error-bars, again obtained using 200 bootstrap samples, are shown for the red and blue galaxies, separately. The solid lines indicate the best-fit parameterizations (equations [13] to [16) for red galaxies. Results shown are for halo masses $M_{S}$ only.

1- $\sigma$ level. Again, since our halo masses are based on the ranking of either $L_{19.5}$ or $M_{\text {stellar, we have effectively as- }}$ sumed a one-to-one relation between halo mass and these mass indicators. This can give rise to spurious correlation between $L_{19.5}$ (or $M_{\text {stellar }}$ ) with the stellar mass of the central galaxies, especially for low-mass halos where the stellar content is dominated by the central galaxy. Therefore the values of $\sigma_{c}$ obtained, especially for halos with $M_{h} \lesssim 10^{13} h^{-1} \mathrm{M}_{\odot}$, may be underestimated and should be considered as lower limits.

For reference, Table 4 lists the average values of the CSMF fitting parameters obtained from the combinations of Samples II and III and group masses $M_{L}$ and $M_{S}$. The error-bars indicate the scatter among these four samples or the scatter obtained from 200 bootstrap samples, whichever is larger (generally the former).

\section{THE GROUP LUMINOSITY AND STELLAR-MASS FUNCTIONS}

In this section, we present our results on the group luminosity and stellar-mass functions. These two functions depend the total luminosity and stellar mass in galaxy groups, and are arguably better suited for comparison with model predictions, as the details about how the total luminosity and total stellar mass are partitioned into individual member galaxies is not important here. We measure the group luminosity and stellar-mass functions for both samples II and III. As mentioned above, these two samples represent two extremes, as far as fibercollision effects are concerned. The results presented in the following are based on the average of samples II and III (evenly weighted), and the error-bars are obtained from the difference between the two samples, or from 200 bootstrap samples for samples II and III, whichever (typically the difference between the two samples) is larger. 


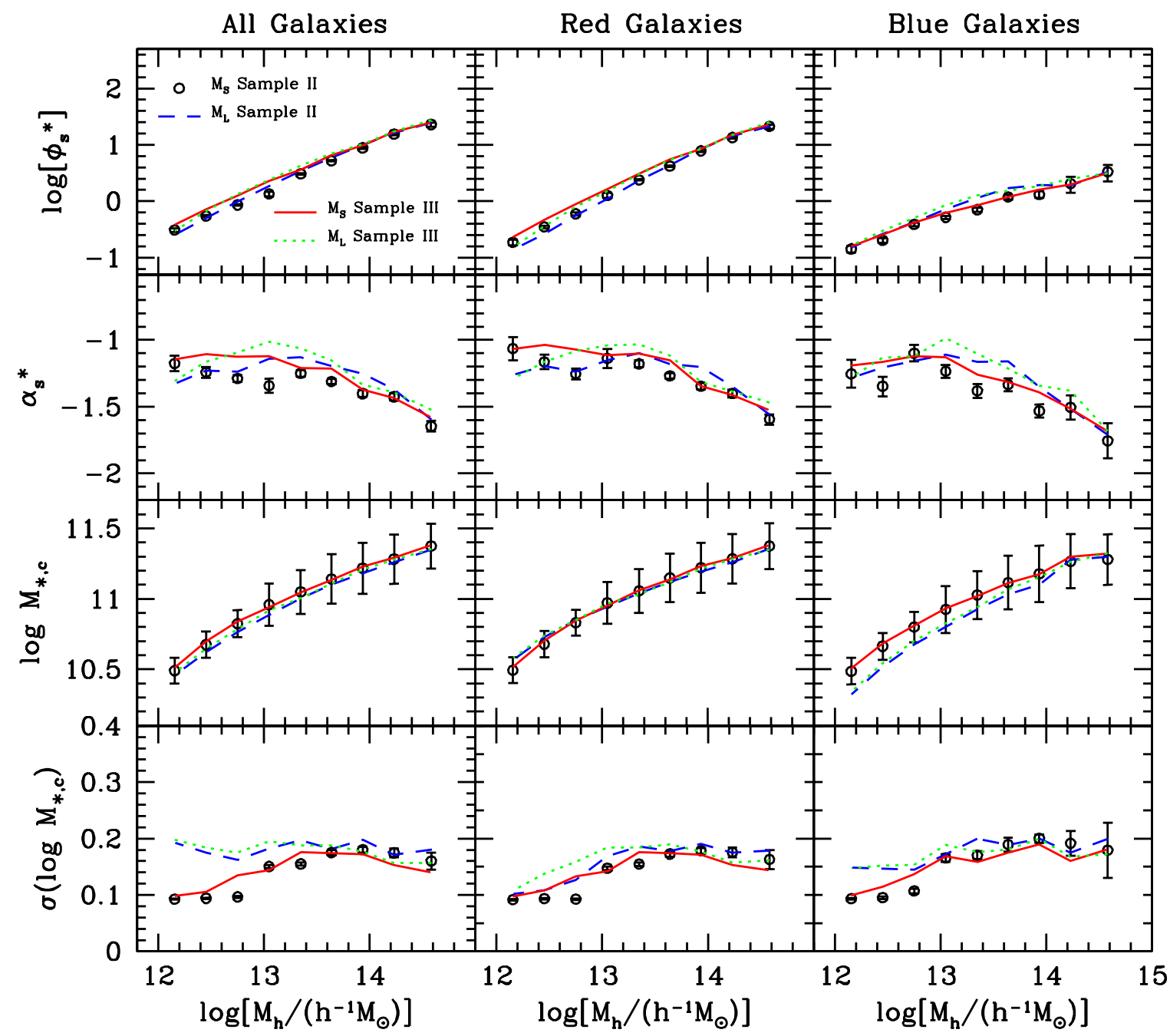

FIG. 6. - The best fit parameters ( $\phi_{s}^{\star}$ upper row, $\alpha_{s}^{\star}$ second row, $M_{*, c}$ third row, and $\sigma_{c}$ bottom row) to the CSMFs shown in Figs. 4 and 5 as functions of halo mass. Panels on the left, in the middle, and on the right show results for all, red, and blue galaxies, respectively. Since we have two different halo mass estimators $\left(M_{S}\right.$ and $\left.M_{L}\right)$ and two main group samples (II and III), we have obtained CLFs for four different combinations of sample and group mass estimator. The results for all four combinations are shown using different symbols and line-styles, as indicated. The error-bars in the first two and last rows indicate the $1-\sigma$ variances obtained from our 200 bootstrap samples. In the third row of panels, however, the error-bars correspond to the log-normal scatter, $\sigma_{c}$, shown in the bottom row of panels. For clarity the error-bars are only shown for the ' $M_{S}$-sample II' case, but they are very similar for the other four cases.

As discussed in Section 2.2, the group catalogue suffers from incompleteness, in that groups within certain luminosity $\left(L_{19.5}\right)$, stellar mass $\left(M_{\text {stellar }}\right)$ and halo mass bins are only complete to certain redshifts. Using the same procedure as described in Section 2.2, we estimate, for each group luminosity $L_{19.5}$ (or stellar mass $M_{\text {stellar }}$ ), the maximum redshift, $z_{\max }$, that satisfies Eq. (8). The resulting $n\left(z_{\max }\right)$ as a function of group luminosity $L_{19.5}$ or stellar mass $M_{\text {stellar }}$ gives, the group luminosity function $\Phi\left(L_{19.5}\right)$ or the group stellar mass function $\Phi\left(M_{\text {stellar }}\right)$, respectively. The results are shown as the solid histograms in the upper panels of Fig. 7.

Note that the group characteristic luminosity $L_{19.5}$ and stellar mass $M_{\text {stellar }}$ are defined to be the total luminosity and stellar mass of member galaxies with ${ }^{0.1} \mathrm{M}_{r}-5 \log h \leq-19.5$. There are certainly groups in which all member galaxies have ${ }^{0.1} \mathrm{M}_{r}-5 \log h>-19.5$, so that $L_{19.5}=0$ and $M_{\text {stellar }}=0$. For these groups we measure their total luminosity $L_{\text {total }}$ and total stellar mass $M_{\text {total }}$ based on all member galaxies that are observed. We measure the luminosity function $\Phi\left(L_{\text {total }}\right)$ and stellar mass function $\Phi\left(M_{\text {total }}\right)$ for these groups using the same method as for $\Phi\left(L_{19.5}\right)$ and $\Phi\left(M_{\text {stellar }}\right)$, i.e., by measuring the $n\left(z_{\max }\right)$. The results are shown in the upper panels of Fig $[7$ as the dotted histograms. For comparison, we also show as the dashed lines the galaxy luminosity function (stellar mass function) for central galaxies that we obtained in Section 3 . The agreement between the group luminosity function $\Phi\left(L_{\text {total }}\right)$ (stellar mass function $\Phi\left(M_{\text {total }}\right)$ ) and the central galaxy luminosity function (stellar mass function) at the faint (low-mass) end is excellent. This simply reflects that the total luminosity $L_{\text {total }}$ and total stellar mass $M_{\text {total }}$ of small groups are dominated by their central galaxies (e.g. Lin \& Mohr 2004; Hansen et al. 2007; Yang et al. 2008b), and implies that missing faint satellites in low mass groups has no significant impact on our estimates of the group luminosity and stellar-mass functions, unless there is a sharp upturn in the conditional luminosity (or stellar mass) function of satellite galaxies, as indicated in 
TABLE 4

The Best Fit Parameters of the CSMFs For ALL, RED AND Blue Galaxies

\begin{tabular}{|c|c|c|c|c|c|c|}
\hline Galaxy type & $\overline{\log \left[M_{h}\right]}$ & $\overline{l o g}\left\langle\left[M_{h}\right]\right\rangle$ & $\phi_{s}^{*}$ & $\alpha_{s}^{*}$ & $\overline{\log M_{*, c}}$ & $\overline{\sigma_{c}}$ \\
\hline (1) & (2) & (3) & (4) & (5) & (6) & (7) \\
\hline ALL & $\begin{array}{l}{[14.40,15.00)} \\
{[14.10,14.40)} \\
{[13.80,14.10)} \\
{[13.50,13.80)} \\
{[13.20,13.50)} \\
{[12.90,13.20)} \\
{[12.60,12.90)} \\
{[12.30,12.60)} \\
{[12.00,12.30)} \\
\end{array}$ & \begin{tabular}{l|}
14.58 \\
14.23 \\
13.94 \\
13.64 \\
13.35 \\
13.05 \\
12.75 \\
12.45 \\
12.16 \\
\end{tabular} & $\begin{array}{c}24.91 \pm 2.51 \\
16.49 \pm 0.79 \\
9.72 \pm 0.63 \\
6.15 \pm 0.77 \\
3.61 \pm 0.52 \\
1.96 \pm 0.47 \\
1.10 \pm 0.22 \\
0.61 \pm 0.10 \\
0.31 \pm 0.06 \\
\end{array}$ & $\begin{array}{l}-1.59 \pm 0.05 \\
-1.40 \pm 0.03 \\
-1.34 \pm 0.07 \\
-1.22 \pm 0.07 \\
-1.16 \pm 0.08 \\
-1.15 \pm 0.14 \\
-1.19 \pm 0.09 \\
-1.19 \pm 0.06 \\
-1.24 \pm 0.09 \\
\end{array}$ & $\begin{array}{l}11.364 \pm 0.018 \\
11.277 \pm 0.013 \\
11.209 \pm 0.020 \\
11.122 \pm 0.020 \\
11.026 \pm 0.024 \\
10.926 \pm 0.032 \\
10.803 \pm 0.034 \\
10.660 \pm 0.033 \\
10.485 \pm 0.022 \\
\end{array}$ & $\begin{array}{l}0.159 \pm 0.017 \\
0.164 \pm 0.011 \\
0.182 \pm 0.011 \\
0.180 \pm 0.007 \\
0.179 \pm 0.018 \\
0.168 \pm 0.025 \\
0.142 \pm 0.035 \\
0.140 \pm 0.046 \\
0.145 \pm 0.058 \\
\end{array}$ \\
\hline RED & $\begin{array}{l}{[14.40,15.00)} \\
{[14.10,14.40)} \\
{[13.80,14.10)} \\
{[13.50,13.80)} \\
{[13.20,13.50)} \\
{[12.90,13.20)} \\
{[12.60,12.90)} \\
{[12.30,12.60)} \\
{[12.00,12.30)}\end{array}$ & $\begin{array}{l}14.58 \\
14.23 \\
13.94 \\
13.64 \\
13.35 \\
13.05 \\
12.75 \\
12.45 \\
12.16\end{array}$ & $\begin{array}{c}22.64 \pm 2.66 \\
14.40 \pm 0.74 \\
8.30 \pm 0.33 \\
4.86 \pm 0.73 \\
2.71 \pm 0.39 \\
1.37 \pm 0.24 \\
0.71 \pm 0.15 \\
0.36 \pm 0.09 \\
0.18 \pm 0.04\end{array}$ & $\begin{array}{l}-1.54 \pm 0.05 \\
-1.39 \pm 0.03 \\
-1.30 \pm 0.07 \\
-1.18 \pm 0.06 \\
-1.10 \pm 0.06 \\
-1.11 \pm 0.07 \\
-1.16 \pm 0.10 \\
-1.14 \pm 0.07 \\
-1.17 \pm 0.12\end{array}$ & $\begin{array}{l}11.365 \pm 0.020 \\
11.278 \pm 0.013 \\
11.216 \pm 0.017 \\
11.131 \pm 0.015 \\
11.047 \pm 0.014 \\
10.958 \pm 0.012 \\
10.844 \pm 0.009 \\
10.713 \pm 0.027 \\
10.539 \pm 0.042\end{array}$ & $\begin{array}{l}0.162 \pm 0.018 \\
0.165 \pm 0.012 \\
0.179 \pm 0.008 \\
0.179 \pm 0.008 \\
0.175 \pm 0.015 \\
0.161 \pm 0.020 \\
0.127 \pm 0.027 \\
0.112 \pm 0.018 \\
0.100 \pm 0.007\end{array}$ \\
\hline BLUE & $\begin{array}{l}{[14.40,15.00)} \\
{[14.10,14.40)} \\
{[13.80,14.10)} \\
{[13.50,13.80)} \\
{[13.20,13.50)} \\
{[12.90,13.20)} \\
{[12.60,12.90)} \\
{[12.30,12.60)} \\
{[12.00,12.30)}\end{array}$ & $\begin{array}{l}14.58 \\
14.23 \\
13.94 \\
13.64 \\
13.35 \\
13.05 \\
12.75 \\
12.45 \\
12.16\end{array}$ & $\begin{array}{l}3.23 \pm 5.24 \\
2.11 \pm 1.06 \\
1.67 \pm 0.27 \\
1.41 \pm 0.26 \\
0.99 \pm 0.26 \\
0.68 \pm 0.14 \\
0.43 \pm 0.05 \\
0.26 \pm 0.04 \\
0.15 \pm 0.03\end{array}$ & $\begin{array}{l}-1.71 \pm 0.31 \\
-1.48 \pm 0.18 \\
-1.41 \pm 0.09 \\
-1.26 \pm 0.08 \\
-1.23 \pm 0.12 \\
-1.12 \pm 0.10 \\
-1.13 \pm 0.07 \\
-1.21 \pm 0.10 \\
-1.25 \pm 0.14\end{array}$ & $\begin{array}{l}11.305 \pm 0.134 \\
11.276 \pm 0.073 \\
11.152 \pm 0.035 \\
11.082 \pm 0.040 \\
10.978 \pm 0.053 \\
10.872 \pm 0.067 \\
10.746 \pm 0.069 \\
10.601 \pm 0.085 \\
10.414 \pm 0.097\end{array}$ & $\begin{array}{l}0.183 \pm 0.049 \\
0.174 \pm 0.044 \\
0.197 \pm 0.013 \\
0.183 \pm 0.012 \\
0.176 \pm 0.017 \\
0.174 \pm 0.010 \\
0.135 \pm 0.020 \\
0.127 \pm 0.027 \\
0.122 \pm 0.030\end{array}$ \\
\hline
\end{tabular}

Note. - Column (1): galaxy type. Column (2): halo mass range. Column (3): average of the logarithm of the halo mass. Column (4)-(7): average of the best fit free parameters to the four measurements of the CSMFs, as shown in Fig. 6 The errors indicate the scatter among these four measurements or the scatter obtained from the 200 bootstrap samples, whichever is larger.

some observations (e.g. Popesso et al. 2006). Thus the overall group luminosity function (stellar mass function) from small to large groups can be obtained by sum up $\Phi\left(L_{19.5}\right)$ and $\Phi\left(L_{\text {total }}\right),\left(\Phi\left(M_{\text {stellar }}\right)\right.$ and $\left.\Phi\left(M_{\text {total }}\right)\right)$. The results are shown as the open circles in the upper panels.

As a further test of the reliability of our results at the low-luminosity and low-mass end, we use another characteristic group luminosity $L_{18.5}$ and another characteristic stellar mass $M_{18.5}$ to represent the luminosity and stellar mass of groups. Here $L_{18.5}\left(M_{18.5}\right)$ is defined to be the total luminosity (stellar mass) of all group members with ${ }^{0.1} \mathrm{M}_{r}-5 \log h \leq-18.5$. Because of the survey apparent magnitude limit, a galaxy fainter than ${ }^{0.1} \mathrm{M}_{r}-5 \log h=-18.5$ is not observed if its redshift is larger than $\sim 0.06$. To avoid such incompleteness, $L_{18.5}$ and $M_{18.5}$ are only measured for groups with redshift $z \leq 0.06$. Our method to estimate $\Phi\left(L_{18.5}\right)$ and $\Phi\left(M_{18.5}\right)$ is the same as that used for $\Phi\left(L_{19.5}\right)$ and $\Phi\left(M_{\text {stellar }}\right)$, except that $z_{\max }$ starts from 0.06 instead of $z=0.2$. The results for $\Phi\left(L_{18.5}\right)$ and $\Phi\left(M_{18.5}\right)$ are shown in the lower two panels of Fig. 7 as the solid histograms. For comparison, we also show $L_{19.5}$ and $M_{\text {stellar }}$ in the corresponding panels as open circles. Clearly, there is a good agreement between $\Phi\left(L_{18.5}\right)$ and $\Phi\left(L_{19.5}\right)$, and between $\Phi\left(M_{18.5}\right)$ and $\Phi\left(M_{\text {stellar }}\right)$ for small groups, suggesting again that our results for low-mass groups are reliable. For massive groups, there is some difference between the two estimates. Unfortunately, the number of such groups is quite small in the volume corresponding to $z=0.06$, and the results for $\Phi\left(L_{18.5}\right)$ and $\Phi\left(M_{18.5}\right)$ are quite uncertain at the massive end. For reference, the data for the group luminosity functions and stellar mass functions shown in Fig. 7 are listed in Table 5 .

Since both the luminosity function and stellar mass function for groups show double power-law behavior, we use the following forms to fit the data:

$$
\Phi\left(L_{19.5}\right)=\Phi_{0} \frac{\left(L_{19.5} / L_{0}\right)^{\alpha}}{\left(x_{0}+\left(L_{19.5} / L_{0}\right)^{4}\right)^{\beta}},
$$

and

$$
\Phi\left(M_{\text {stellar }}\right)=\Phi_{0} \frac{\left(M_{\text {stellar }} / M_{0}\right)^{\alpha}}{\left(x_{0}+\left(M_{\text {stellar }} / M_{0}\right)^{4}\right)^{\beta}} .
$$

The best fit results are shown in the lower two panels of Fig. 7 as the long-dashed lines. The corresponding best fit parameters are $\left[\Phi_{0}, \log L_{0}, x_{0}, \alpha, \beta\right]=[0.00580$, $10.30,0.1786,-0.2226,0.4236]$ and $\left[\Phi_{0}, \log M_{0}, x_{0}, \alpha, \beta\right]$ $=[0.00731,10.67,0.7243,-0.2229,0.3874]$, for the luminosity and stellar-mass functions, respectively. Note that all the group luminosity functions and stellar mass functions are calculated in terms of $h^{3} \mathrm{Mpc}^{-3} \mathrm{~d} \log L$ and $h^{3} \mathrm{Mpc}^{-3} \mathrm{~d} \log M_{*}$ ), where $\log$ is the 10 based logarithm.

It is interesting to compare the group luminosity and stellar-mass functions with the halo mass function predicted by the current CDM model of structure forma- 

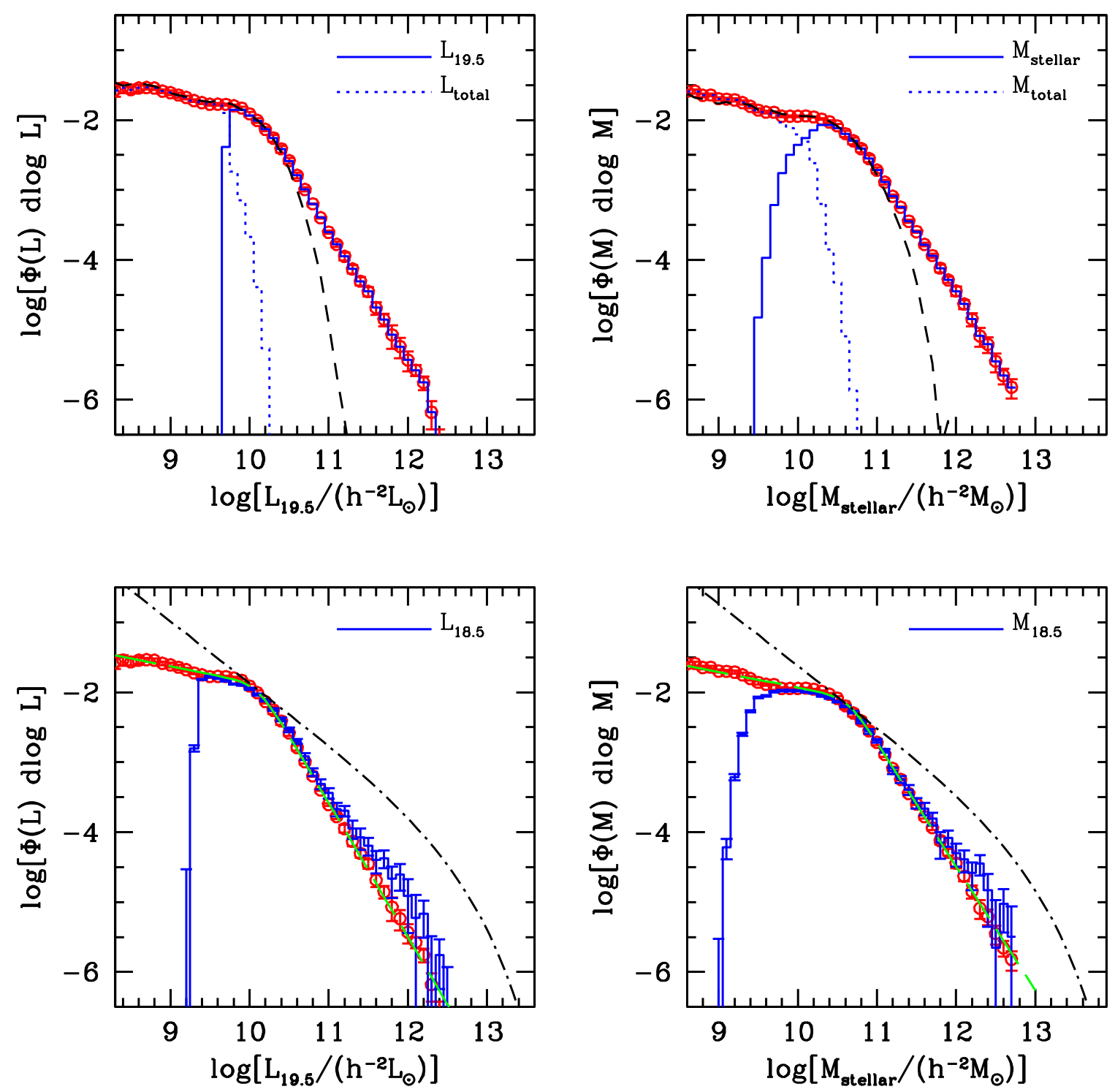

FIG. 7.- The group luminosity functions and stellar mass functions. The upper-left panel: solid and dotted histograms are the group luminosity functions for $L_{19.5}$ and $L_{\text {total }}$, respectively. The open circles with error-bars are the sum of the two contributions. The dashed line is the luminosity function for central galaxies shown in Fig. 2 The upper-right panel: solid and dotted histograms are the group stellar mass functions for $M_{\text {stellar }}$ and $M_{\text {total }}$, respectively. The open circles with error-bars are the sum of the two contributions. The dashed line is the stellar mass function for central galaxies shown in Fig. 2 The lower-left panel: the open circles with error-bars are the same as those in the upper-left panel and the long dashed line shows the best fitting results. The solid histogram is the group luminosity function for $L_{18.5}$. The lower-right panel: the open circles with error-bars are the same as those in the upper-right panel and the long dashed line shows the best fitting results. The solid histogram is the group stellar mass function for $M_{18.5}$. See text for the definitions of $L_{19.5}, L_{\text {total }}$, $L_{18.5}, M_{\text {stellar }}, M_{\text {total }}$ and $M_{18.5}$. For comparison, in the lower two panels, we show as dot-dashed lines the scaled halo mass function (Warren et al. 2006) for the same $\Lambda$ CDM 'concordance' cosmology.

tion. In the lower-left panel of Fig 7 we plot, as the dot-dashed line, the halo mass function which is scaled with a (minimum) constant mass-to-light ratio, $M_{h} / L_{19.5}=47 h \mathrm{M}_{\odot} / \mathrm{L}_{\odot}$, so that the halo mass function touches the group luminosity function at $\log L_{19.5} \sim 10.2$. Here the halo mass function is estimated using the Warren et al. (2006) model and adopting the $\Lambda$ CDM 'concordance' cosmology with parameters listed at the end of Section 1. Clearly, the scaled halo mass function has a very different shape from the observed group luminosity function, indicating that the mass-to-light ratio must depend strongly on halo mass. The mass-tolight ratio reaches its minimum in groups (halos) with $\log L_{19.5} \sim 10.2$, and is larger for both smaller and big- ger halos. In the lower-right panel of Fig. 7 we compare the group stellar mass function (open circles) with the halo mass function scaled with a constant halo-tostellar mass ratio (dot-dashed line), $M_{h} / M_{\text {stellar }}=25 \mathrm{~h}$. Here the halo-to-stellar mass ratio reaches its minimum in groups (halos) with $\log M_{\text {stellar }} \sim 10.6$, corresponding to halos with masses $M_{h} \sim 11.9$ (almost exactly the same halo mass that the group mass-to-light ratio reaches its minimum), suggesting that star formation efficiency is the highest in such halos. These results are in excellent agreement with those obtained from the clustering properties of galaxies using the CLF and HOD formalism (Yang et al. 2003; Tinker et al 2005; van den Bosch et al. 2007; Cacciato et al. 2008), and at the relative massive 
TABLE 5

THE GROUP LUMINOSITY FUNCTIONS AND STELLAR MASS FUNCTIONS

\begin{tabular}{|c|c|c|c|c|c|c|}
\hline $\log L\left(\log M_{*}\right)$ & $\Phi\left(L_{\text {total }}\right)$ & $\Phi\left(L_{19.5}\right)$ & $\Phi\left(L_{18.5}\right)$ & $\Phi\left(M_{\text {total }}\right)$ & $\Phi\left(M_{\text {stellar }}\right)$ & $\Phi\left(M_{18.5}\right)$ \\
\hline (1) & (2) & (3) & (4) & (5) & (6) & (7) \\
\hline 8.1 & $3.2253 \pm 0.5322$ & $0.0000 \pm 0.0000$ & $0.0000 \pm 0.0000$ & $0.0000 \pm 0.0000$ & $0.0000 \pm 0.0000$ & $0.0000 \pm 0.0000$ \\
\hline 8.2 & $3.4201 \pm 0.6087$ & $0.0000 \pm 0.0000$ & $0.0000 \pm 0.0000$ & $0.0000 \pm 0.0000$ & $0.0000 \pm 0.0000$ & $0.0000 \pm 0.0000$ \\
\hline 8.3 & $2.6641 \pm 0.5050$ & $0.0000 \pm 0.0000$ & $0.0000 \pm 0.0000$ & $0.0000 \pm 0.0000$ & $0.0000 \pm 0.0000$ & $0.0000 \pm 0.0000$ \\
\hline 8.4 & $2.8979 \pm 0.4862$ & $0.0000 \pm 0.0000$ & $0.0000 \pm 0.0000$ & $2.3283 \pm 0.4981$ & $0.0000 \pm 0.0000$ & $0.0000 \pm 0.0000$ \\
\hline 8.5 & $2.7062 \pm 0.2931$ & $0.0000 \pm 0.0000$ & $0.0000 \pm 0.0000$ & $2.2944 \pm 0.3343$ & $0.0000 \pm 0.0000$ & $0.0000 \pm 0.0000$ \\
\hline 8.6 & $2.8626 \pm 0.3414$ & $0.0000 \pm 0.0000$ & $0.0000 \pm 0.0000$ & $2.5619 \pm 0.1969$ & $0.0000 \pm 0.0000$ & $0.0000 \pm 0.0000$ \\
\hline 8.7 & $2.9337 \pm 0.1897$ & $0.0000 \pm 0.0000$ & $0.0000 \pm 0.0000$ & $2.5771 \pm 0.1779$ & $0.0000 \pm 0.0000$ & $0.0000 \pm 0.0000$ \\
\hline 8.8 & $2.8651 \pm 0.1770$ & $0.0000 \pm 0.0000$ & $0.0000 \pm 0.0000$ & $2.2581 \pm 0.1293$ & $0.0000 \pm 0.0000$ & $0.0000 \pm 0.0000$ \\
\hline 8.9 & $2.5868 \pm 0.1677$ & $0.0000 \pm 0.0000$ & $0.0000 \pm 0.0000$ & $2.2786 \pm 0.1022$ & $0.0000 \pm 0.0000$ & $0.0000 \pm 0.0000$ \\
\hline 9.0 & $2.4589 \pm 0.2062$ & $0.0000 \pm 0.0000$ & $0.0000 \pm 0.0000$ & $2.0151 \pm 0.0913$ & $0.0000 \pm 0.0000$ & $0.0000 \pm 0.0003$ \\
\hline 9.1 & $2.2798 \pm 0.1883$ & $0.0000 \pm 0.0000$ & $0.0000 \pm 0.0000$ & $1.9811 \pm 0.1045$ & $0.0000 \pm 0.0000$ & $0.0060 \pm 0.0020$ \\
\hline 9.2 & $2.1088 \pm 0.1361$ & $0.0000 \pm 0.0000$ & $0.0000 \pm 0.0030$ & $1.9434 \pm 0.1014$ & $0.0000 \pm 0.0000$ & $0.0613 \pm 0.0065$ \\
\hline 9.3 & $1.8898 \pm 0.0820$ & $0.0000 \pm 0.0000$ & $0.1574 \pm 0.0163$ & $1.7766 \pm 0.1248$ & $0.0000 \pm 0.0000$ & $0.2513 \pm 0.0117$ \\
\hline 9.4 & $1.7805 \pm 0.1027$ & $0.0000 \pm 0.0000$ & $1.5209 \pm 0.0291$ & $1.5740 \pm 0.1087$ & $0.0000 \pm 0.0004$ & $0.5356 \pm 0.0192$ \\
\hline 9.5 & $1.6742 \pm 0.0629$ & $0.0000 \pm 0.0000$ & $1.6765 \pm 0.0450$ & $1.3785 \pm 0.0983$ & $0.0015 \pm 0.0028$ & $0.8491 \pm 0.0229$ \\
\hline 9.6 & $1.6838 \pm 0.2660$ & $0.0000 \pm 0.1849$ & $1.5925 \pm 0.0407$ & $1.2934 \pm 0.0800$ & $0.0107 \pm 0.0160$ & $0.8836 \pm 0.0238$ \\
\hline 9.7 & $1.2575 \pm 0.2790$ & $0.4140 \pm 0.2468$ & $1.4683 \pm 0.0367$ & $1.2237 \pm 0.0737$ & $0.0610 \pm 0.0349$ & $1.0167 \pm 0.0220$ \\
\hline 9.8 & $0.1845 \pm 0.2831$ & $1.4048 \pm 0.2795$ & $1.3138 \pm 0.0266$ & $0.9211 \pm 0.0728$ & $0.1742 \pm 0.0574$ & $1.0525 \pm 0.0230$ \\
\hline 9.9 & $0.0713 \pm 0.0668$ & $1.3972 \pm 0.1135$ & $1.2828 \pm 0.0312$ & $0.7662 \pm 0.0597$ & $0.3168 \pm 0.0601$ & $1.0576 \pm 0.0227$ \\
\hline 10.0 & $0.0213 \pm 0.0209$ & $1.1648 \pm 0.0569$ & $1.1205 \pm 0.0360$ & $0.6207 \pm 0.0561$ & $0.4414 \pm 0.0561$ & $1.0479 \pm 0.0220$ \\
\hline 10.1 & $0.0041 \pm 0.0025$ & $0.9810 \pm 0.0490$ & $0.9278 \pm 0.0323$ & $0.4901 \pm 0.0900$ & $0.5571 \pm 0.0562$ & $1.0148 \pm 0.0185$ \\
\hline 10.2 & $0.0005 \pm 0.0014$ & $0.7398 \pm 0.0543$ & $0.7652 \pm 0.0244$ & $0.2426 \pm 0.0767$ & $0.7160 \pm 0.0428$ & $0.9849 \pm 0.0218$ \\
\hline 10.3 & $0.0000 \pm 0.0001$ & $0.5534 \pm 0.0597$ & $0.5713 \pm 0.0254$ & $0.0630 \pm 0.0526$ & $0.8471 \pm 0.0456$ & $0.8950 \pm 0.0262$ \\
\hline 10.4 & $0.0000 \pm 0.0000$ & $0.3862 \pm 0.0426$ & $0.4079 \pm 0.0239$ & $0.0140 \pm 0.0097$ & $0.8444 \pm 0.0435$ & $0.8355 \pm 0.0267$ \\
\hline 10.5 & $0.0000 \pm 0.0000$ & $0.2608 \pm 0.0242$ & $0.2914 \pm 0.0242$ & $0.0048 \pm 0.0007$ & $0.7618 \pm 0.0621$ & $0.7352 \pm 0.0218$ \\
\hline 10.6 & $0.0000 \pm 0.0000$ & $0.1620 \pm 0.0140$ & $0.1987 \pm 0.0145$ & $0.0008 \pm 0.0005$ & $0.6367 \pm 0.0635$ & $0.6321 \pm 0.0159$ \\
\hline 10.7 & $0.0000 \pm 0.0000$ & $0.1020 \pm 0.0069$ & $0.1215 \pm 0.0131$ & $0.0001 \pm 0.0003$ & $0.5056 \pm 0.0562$ & $0.5197 \pm 0.0201$ \\
\hline 10.8 & $0.0000 \pm 0.0000$ & $0.0635 \pm 0.0012$ & $0.0731 \pm 0.0094$ & $0.0000 \pm 0.0001$ & $0.3893 \pm 0.0433$ & $0.3832 \pm 0.0225$ \\
\hline 10.9 & $0.0000 \pm 0.0000$ & $0.0400 \pm 0.0008$ & $0.0488 \pm 0.0078$ & $0.0000 \pm 0.0000$ & $0.2816 \pm 0.0307$ & $0.2774 \pm 0.0203$ \\
\hline 11.0 & $0.0000 \pm 0.0000$ & $0.0249 \pm 0.0011$ & $0.0363 \pm 0.0062$ & $0.0000 \pm 0.0000$ & $0.1918 \pm 0.0183$ & $0.2025 \pm 0.0127$ \\
\hline 11.1 & $0.0000 \pm 0.0000$ & $0.0167 \pm 0.0015$ & $0.0215 \pm 0.0048$ & $0.0000 \pm 0.0000$ & $0.1295 \pm 0.0106$ & $0.1460 \pm 0.0079$ \\
\hline 11.2 & $0.0000 \pm 0.0000$ & $0.0112 \pm 0.0014$ & $0.0202 \pm 0.0033$ & $0.0000 \pm 0.0000$ & $0.0817 \pm 0.0048$ & $0.0751 \pm 0.0079$ \\
\hline 11.3 & $0.0000 \pm 0.0000$ & $0.0074 \pm 0.0011$ & $0.0113 \pm 0.0033$ & $0.0000 \pm 0.0000$ & $0.0566 \pm 0.0019$ & $0.0568 \pm 0.0066$ \\
\hline 11.4 & $0.0000 \pm 0.0000$ & $0.0050 \pm 0.0007$ & $0.0084 \pm 0.0028$ & $0.0000 \pm 0.0000$ & $0.0358 \pm 0.0016$ & $0.0404 \pm 0.0067$ \\
\hline 11.5 & $0.0000 \pm 0.0000$ & $0.0035 \pm 0.0006$ & $0.0064 \pm 0.0018$ & $0.0000 \pm 0.0000$ & $0.0253 \pm 0.0007$ & $0.0263 \pm 0.0044$ \\
\hline 11.6 & $0.0000 \pm 0.0000$ & $0.0021 \pm 0.0004$ & $0.0040 \pm 0.0014$ & $0.0000 \pm 0.0000$ & $0.0166 \pm 0.0009$ & $0.0218 \pm 0.0046$ \\
\hline 11.7 & $0.0000 \pm 0.0000$ & $0.0014 \pm 0.0003$ & $0.0043 \pm 0.0012$ & $0.0000 \pm 0.0000$ & $0.0115 \pm 0.0008$ & $0.0158 \pm 0.0032$ \\
\hline 11.8 & $0.0000 \pm 0.0000$ & $0.0008 \pm 0.0003$ & $0.0022 \pm 0.0010$ & $0.0000 \pm 0.0000$ & $0.0076 \pm 0.0010$ & $0.0071 \pm 0.0030$ \\
\hline 11.9 & $0.0000 \pm 0.0000$ & $0.0006 \pm 0.0002$ & $0.0025 \pm 0.0010$ & $0.0000 \pm 0.0000$ & $0.0052 \pm 0.0005$ & $0.0083 \pm 0.0022$ \\
\hline 12.0 & $0.0000 \pm 0.0000$ & $0.0004 \pm 0.0001$ & $0.0012 \pm 0.0009$ & $0.0000 \pm 0.0000$ & $0.0036 \pm 0.0005$ & $0.0050 \pm 0.0018$ \\
\hline 12.1 & $0.0000 \pm 0.0000$ & $0.0003 \pm 0.0000$ & $0.0006 \pm 0.0007$ & $0.0000 \pm 0.0000$ & $0.0023 \pm 0.0003$ & $0.0041 \pm 0.0014$ \\
\hline 12.2 & $0.0000 \pm 0.0000$ & $0.0002 \pm 0.0000$ & $0.0007 \pm 0.0004$ & $0.0000 \pm 0.0000$ & $0.0014 \pm 0.0003$ & $0.0026 \pm 0.0011$ \\
\hline 12.3 & $0.0000 \pm 0.0000$ & $0.0001 \pm 0.0000$ & $0.0000 \pm 0.0003$ & $0.0000 \pm 0.0000$ & $0.0008 \pm 0.0002$ & $0.0035 \pm 0.0012$ \\
\hline 12.4 & $0.0000 \pm 0.0000$ & $0.0000 \pm 0.0000$ & $0.0002 \pm 0.0001$ & $0.0000 \pm 0.0000$ & $0.0006 \pm 0.0002$ & $0.0015 \pm 0.0010$ \\
\hline 12.5 & $0.0000 \pm 0.0000$ & $0.0000 \pm 0.0000$ & $0.0000 \pm 0.0001$ & $0.0000 \pm 0.0000$ & $0.0004 \pm 0.0001$ & $0.0002 \pm 0.0008$ \\
\hline 12.6 & $0.0000 \pm 0.0000$ & $0.0000 \pm 0.0000$ & $0.0000 \pm 0.0000$ & $0.0000 \pm 0.0000$ & $0.0002 \pm 0.0001$ & $0.0010 \pm 0.0006$ \\
\hline 12.7 & $0.0000 \pm 0.0000$ & $0.0000 \pm 0.0000$ & $0.0000 \pm 0.0000$ & $0.0000 \pm 0.0000$ & $0.0002 \pm 0.0000$ & $0.0003 \pm 0.0006$ \\
\hline
\end{tabular}

Note. - Column (1): the median of the logarithm of the group luminosity or stellar mass with bin width $\Delta \log L=0.05$ (or $\left.\Delta \log M_{*}=0.05\right)$. Column (2): the average group luminosity function for $L_{\text {total }}$, where $L_{\text {total }}$ is the total luminosity of all group members in which the central galaxy has luminosity ${ }^{0.1} \mathrm{M}_{r}-5 \log h>-19.5$. Column (3): the average group luminosity function for $L_{19.5}$, where $L_{19.5}$ is the luminosity of all group members with ${ }^{0.1} \mathrm{M}_{r}-5 \log h \leq-19.5$. Column (4) the average group luminosity function for $L_{18.5}$, where $L_{18.5}$ is the total luminosity of all group members with ${ }^{0.1} \mathrm{M}_{r}-5 \log h \leq-18.5$. Columns (5)-(7): Similar to Columns (2)-(4) but for the average stellar mass functions. In this table, the averages are obtained from the two measurements of the luminosity functions or stellar mass functions from samples II and III, respectively. The error indicates the scatter among these two measurements or the scatter obtained from 200 bootstrap samples, whichever is larger. Note that all the group luminosity (stellar mass) functions listed in this table are calculated in units of $10^{-2} h^{3} \mathrm{Mpc}^{-3} \mathrm{~d} \log L$ (or $10^{-2} h^{3} \mathrm{Mpc}^{-3} \mathrm{~d} \log M_{*}$ ), where $\log$ is the 10 based logarithm.

end with those obtained from weak lensing observations (e.g. Sheldon et al. 2007).

\section{THE CENTRAL GALAXIES IN SMALL HALOS}

In this section we discuss the implication of the observed faint end slope of the central galaxy luminosity and stellar mass functions for the relationship between galaxies and dark matter halos. In Yang et al. (2005b) and Paper II, we have measured the CLFs for relatively massive halos with $M_{h} \gtrsim 10^{12} h^{-1} \mathrm{M}_{\odot}$ directly from group samples. Unfortunately, galaxies in less massive halos are not well studied, partly because the halo masses for small groups are difficult to estimate. Here, with the help of the $L_{c}-M_{h}\left(M_{*, c}-M_{h}\right)$ relations that we obtained for relatively massive halos and using the luminosity functions and stellar mass functions for central galaxies, we can probe the halo properties of low-mass central galaxies in a statistical way. As discussed in Sections 4 and 5 , a small halo is usually dominated by a single central galaxy. The $L_{c}-M_{h}$ and $M_{*, c}-M_{h}$ re- 

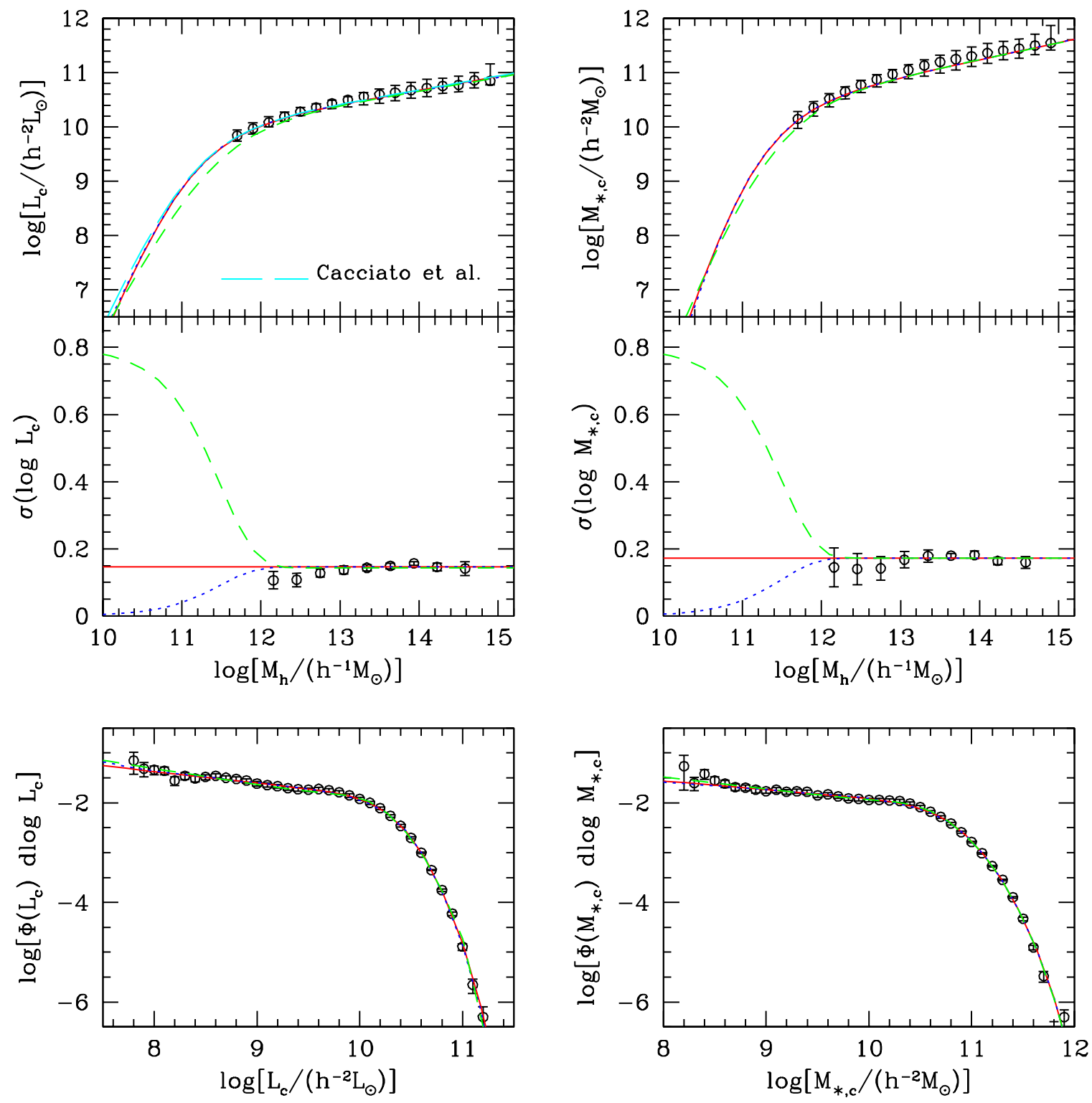

FIG. 8.- The properties of central galaxies: shown in the left and right panels are results for the luminosity $L_{c}$ and stellar mass $M_{*, c}$, respectively. The open circles in each panel are the data we extracted from the SDSS DR4 group catalogue, while the solid, dotted and dashed lines are the best fitting results (see text for details). Upper-left panel: the $L_{c}-M_{h}$ relation, where the data points are obtained from the left panel of Fig. 6 in Paper II. The long-dashed line in this panel illustrates the $L_{c}-M_{h}$ relation predicted by Cacciato et al. (2008). Upper-right panel: the $M_{*, c}-M_{h}$ relation, where the data points are obtained from the right panel of Fig. 6 in Paper II. Middle-left panel: the $\sigma\left(\log L_{c}\right)-M_{h}$ relation, where the data points are obtained from the lower-left panel of Fig. 4 in paper II. Middle-right panel: the $\sigma\left(\log M_{*, c}\right)-M_{h}$ relation, where the data points are obtained from the lower-left panel of Fig. 6. Lower-left panel: the luminosity function of the central galaxies, $\Phi\left(L_{c}\right)$, where the data points are the same as those shown in the middle-left panel of Fig. 2 Lower-right panel: the stellar mass function of central galaxies, $\Phi\left(M_{*, c}\right)$, where the data points are the same as those shown in the middle-right panel of Fig. 2

lations for groups with masses down to $\sim 10^{11.8} h^{-1} \mathrm{M}_{\odot}$ can be obtained directly from the group catalogue, and are shown as open circles with error-bars in the upper panels of Fig. 8. For these relatively massive groups, we can also estimate $\sigma\left(\log L_{c}\right)$ and $\sigma\left(\log M_{*, c}\right)$ as functions of halo mass. These are shown in the middle panels of Fig. 8 as the open circles with error-bars. Finally, the luminosity and stellar mass functions for central galaxies, which have been obtained in Section 3, are repeated in the lower panels of Fig. 8 as open circles with error-bars.

Following paper II, we model the mean $L_{c}-M_{h}$ and
$M_{*, c}-M_{h}$ relations using the following functional forms:

$$
L_{c}=L_{0} \frac{\left(M_{h} / M_{1}\right)^{\alpha+\beta}}{\left(1+M_{h} / M_{1}\right)^{\beta}},
$$

and

$$
M_{*, c}=M_{0} \frac{\left(M_{h} / M_{1}\right)^{\alpha+\beta}}{\left(1+M_{h} / M_{1}\right)^{\beta}},
$$

where $M_{1}$ is a characteristic halo mass so that $L_{c} \propto$ $M_{h}^{\alpha+\beta}\left(M_{*, c} \propto M_{h}^{\alpha+\beta}\right)$ for $M_{h} \ll M_{1}$ and $L_{c} \propto M_{h}^{\alpha}$ $\left(M_{*, c} \propto M_{h}^{\alpha}\right)$ for $M_{h} \gg M_{1}$. Note that the parameters $M_{1}, \alpha$ and $\beta$ may have different values in the two rela- 
tions. Clearly, for bright (massive) central galaxies, these relations are well constrained by the data points shown in the upper panels of Fig. 8. Unfortunately, no such direct constraints are available for the faint (low-mass) centrals. However, an indirect constraint comes from the observed luminosity and stellar mass functions of central galaxies. In general, we can write

$$
\Phi\left(L_{c}\right)=\int_{0}^{\infty} \Phi_{\mathrm{cen}}\left(L \mid M_{h}\right) n\left(M_{h}\right) \mathrm{d} M_{h},
$$

and

$$
\Phi\left(M_{*, c}\right)=\int_{0}^{\infty} \Phi_{\text {cen }}\left(M_{*} \mid M_{h}\right) n\left(M_{h}\right) \mathrm{d} M_{h},
$$

where $n(M)$ is the mass function of dark matter halos, and $\Phi_{\text {cen }}\left(L \mid M_{h}\right)$ and $\Phi_{\text {cen }}\left(M_{*} \mid M_{h}\right)$ are the CLF and CSMF of central galaxies, respectively. If we model both $\Phi_{\text {cen }}\left(L \mid M_{h}\right)$ and $\Phi_{\text {cen }}\left(M_{*} \mid M_{h}\right)$ with a lognormal form, they are completely determined by the mean relations, (19) and (20) and the corresponding dispersions, $\sigma\left(\log L_{c}\right)$ and $\sigma\left(\log M_{*, c}\right)$. As a simple model we first assume these dispersions to be constant: $\sigma\left(\log L_{c}\right)=\sigma_{0}$ and $\sigma\left(\log M_{*, c}\right)=\sigma_{0}$ (again $\sigma_{0}$ may have different values in the two cases). Thus, for each case, we have five free parameters, $L_{0}$ (or $M_{0}$ ), $M_{1}, \alpha, \beta$ and $\sigma_{0}$.

Using the halo mass function predicted by the $\Lambda \mathrm{CDM}$ 'concordance' cosmology, we fit the models described above to the observational data shown in Fig. 8. The fitting is performed with a standard least $\chi^{2}$ algorithm. For the luminosity of the central galaxies, we use $\chi^{2}=$ $\chi^{2}\left(L_{c}\right)+\chi^{2}(\sigma)+\chi^{2}\left(\Phi\left(L_{c}\right)\right)$, and for the stellar mass of the central galaxies, we use $\chi^{2}=\chi^{2}\left(M_{*, c}\right)+\chi^{2}(\sigma)+$ $\chi^{2}\left(\Phi\left(M_{*, c}\right)\right)$. Here, $\chi^{2}\left(L_{c}\right), \chi^{2}(\sigma)$ and $\chi^{2}\left(\Phi\left(L_{c}\right)\right.$ ) (or $\chi^{2}\left(M_{*, c}\right), \chi^{2}(\sigma)$ and $\left.\chi^{2}\left(\Phi\left(M_{*, c}\right)\right)\right)$ are calculated according to the deviations of the model predictions from the observational data shown in the upper-, middle-, and lower-left (or right) panels of Fig. 8. respectively. Based on all the data points shown in the left hand side panels of Fig. 8, we obtain $\log L_{0}=9.9078, \log M_{1}=11.0096$, $\alpha=0.2566, \beta=3.4037, \sigma_{0}=0.1462$. The resulting best fit is shown in each panel as the solid lines. The agreement between the best fit model and the data is remarkably good. The best fitting parameters indicate that $L_{c} \propto M_{h}^{3.7}$ for $\log M_{h} \ll 11.0$, suggesting that the star formation efficiency decreases dramatically in small halos. These results, especially the slopes of the $L_{c}-M_{h}$ relation $(\alpha \sim 0.25$ at the massive end, and $\alpha+\beta>>1$ at the low-mass end), are in good agreement with previous results (e.g., Vale \& Ostriker 2004, 2006; Cooray 2005; Yang et al. 2003; Yang et al. 2005c; van den Bosch et al. 2007; Hansen et al. 2007; Zheng et al. 2007; Popesso et al. 2007; Brough et al. 2008; Conroy \& Wechsler 2008). The physical reason for this change in slope is probably a combination of AGN feedback, and the change in the efficiency of radiative cooling, supernova feedback and dynamical friction (e.g. Lin et al. 2004; Cooray \& Milosavljević 2005). For comparison, we also show in the upper-left panel the mean $L_{c}-M_{h}$ relation obtained by Cacciato et al. (2008) based on the CLF models. The excellent agreement with our prediction indicates that although obtained via different methods, the mean $L_{c}-M_{h}$ relation are well constrained in both investigations.
For the stellar mass of the central galaxies, fitting the model to the data shown in the right panels of Fig. 8 gives $\log M_{0}=10.3061, \log M_{1}=11.0404, \alpha=0.3146$, $\beta=4.5427, \sigma_{0}=0.1730$. Note that $M_{*, c} \propto M_{h}^{4.9}$ for $\log M_{h} \ll 11.0$, which is even steeper than the $L_{c}-M_{h}$ relation.

The above results are obtained under the assumption that the value of the dispersion, $\sigma$, is independent of halo mass. Although consistent with the data for $M_{h} \gtrsim$ $10^{12} h^{-2} \mathrm{M}_{\odot}$, and supported by satellite kinematics and semi-analytical models (More et al. 2008), it is not well constrained in low mass halos. We therefore now test the impact of this assumption on our results. For this purpose, we consider two models where $\sigma$ is required to change from the observed value at $\log \left(M_{h} / h^{-1} \mathrm{M}_{\odot}\right) \sim 12$ either to 0 or to 0.8 at $\log \left(M_{h} / h^{-1} \mathrm{M}_{\odot}\right) \sim 10$ (see the dotted and dashed curves in the middle panels of Fig. 8. respectively). The best fits of these two test models are shown as the dotted and dashed lines in the other panels. Clearly, our results for the $L_{c}-M_{h}$ and $M_{c}-M_{h}$ relations are robust with respect to our assumption that $\sigma$ is independent of halo mass.

\section{SUMMARY}

In this paper, we have derived the luminosity and stellar mass functions for different populations of galaxies (central versus satellite; red versus blue; and galaxies in halos of different masses), and for groups themselves, using a large galaxy group catalogue constructed from the SDSS Data Release 4 (DR4). Our main results can be summarized as follows:

1. For central galaxies, the conditional stellar mass function (CSMF), which describes the stellar mass distribution of galaxies in halos of a given mass can be well described by a log-normal distribution, with a width $\sigma_{\log M_{*}} \sim 0.17$, quite independent of the host halo mass. The median central stellar mass increases rapidly with halo mass, $M_{*} \propto M_{h}^{4.9}$, for halos with masses $M_{h} \ll 10^{11} h^{-1} \mathrm{M}_{\odot}$, but only slowly, $M_{*} \propto M_{h}^{0.3}$, for halos with $M_{h} \gg$ $10^{13} h^{-1} \mathrm{M}_{\odot}$.

2. For satellite galaxies, the conditional stellar mass function in halos of different masses can be described reasonably well by a modified Schechter form than breaks away faster than the Schechter function at the massive end. The faint end slope appears to be steeper for more massive halos. On average, there are about 3 times as many central galaxies as satellites.

3. When stellar mass functions are measured separately for galaxies of different colors, we find that the central population is dominated by red galaxies at the massive end, and by blue galaxies at the lowmass end. Among the satellite population, there are in general more red galaxies than blue ones. At the very low-mass end $\left(M_{*} \lesssim 10^{9} h^{-2} \mathrm{M}_{\odot}\right)$, there is a marked increase in the number of red centrals. We speculate that these galaxies are located close to large halos so that their star formation has been affected by their environments. 
4. The stellar-mass function of galaxy groups, which describes the number density of galaxy groups as a function of the total stellar mass of group member galaxies, is well described by a double power law, with a characteristic stellar mass at $\sim 4 \times 10^{10} h^{-2} \mathrm{M}_{\odot}$. This form is very different from that of the halo mass function, indicating that the efficiencies of star formation in halos of different masses are very different.

5. The stellar mass function for the central galaxies can be used to provide stringent constraint on the mean $M_{*, c}-M_{h}$ relation for low-mass halos.

We anticipate that a comparison of these results with predictions of numerical simulations and/or semianalytical models will provide stringent constraints on how galaxies form and evolve in dark matter halos.

\section{ACKNOWLEDGMENTS}

We are grateful to the anonymous referee for useful and insightful comments that greatly helped to improve the presentation of this paper. XY acknowledges the University of Massachusetts for hospitality where this work was finalized. This work is supported by the One Hundred Talents project, Shanghai Pujiang Program (No. 07pj14102), 973 Program (No. 2007CB815402), the CAS Knowledge Innovation Program (Grant No. KJCX2YW-T05) and grants from NSFC (Nos. 10533030, 10673023, 10821302). HJM would like to acknowledge the support of NSF AST-0607535, NASA AISR-126270 and NSF IIS-0611948.

\section{REFERENCES}

Adelman-McCarthy J.K., et al., 2006, ApJS, 162, 38

Baldry I.K., Glazebrook K., Brinkmann J., Ivezic Z., Lupton R.H., Nichol R.C., Szalay A.S., 2004, ApJ, 600, 681

Baldry I.K., Glazebrook K., Driver S.P., 2008, MNRAS, 388, 945

Bell E.F., McIntosh D.H., Katz N., Weinberg M.D., 2003, ApJS, 149,289

Berlind A.A., Weinberg D.H., 2002, ApJ, 575, 587

Berlind A.A., et al. 2006, ApJS, 167, 1

Berlind A.A., Kazin E., Blanton M.R., Pueblas S., Scoccimarro R., Hogg D.H., 2007, preprint (astro-ph/0610524)

Blanton M. R. et al. , 2003a, AJ, 125, 2348

Blanton M. R. et al. , 2003b, ApJ, 592, 819

Blanton M.R., Eisenstein D.J., Hogg D.W., Schlegel D.J., Brinkmann J., 2005a, ApJ, 629, 143

Blanton M.R. et al. , 2005b, AJ, 129, 2562

Blanton M.R., Roweis S., 2007, AJ, 133, 734

Borch A., et al., 2006, A\&A, 453, 869

Brough S., Couch W.J., Collins C.A., Jarrett T., Burke D.J., Mann R.G., 2008, MNRAS, 385L, 103

Brown M.J.I., et al., 2008, ApJ, 682, 937

Bullock J.S., Wechsler, R.H., Somerville R.S., 2002, MNRAS, 329,246

Cacciato M., van den Bosch F.C., More S., Li R., Mo H.J., Yang X., 2008, MNRAS, submitted (arXiv:0807.4932)

Coil A.L., et al., 2006, ApJ, 638, 668

Colless M., et al., 2001 MNRAS, 328, 1039

Collister A.A., Lahav O., 2005, MNRAS, 361, 415

Conroy C., Wechsler R.H.,., 2008, preprint, arXiv:0805.3346

Cooray A., 2005, MNRAS, 364, 303

Cooray A., Milosavljević M., 2005, ApJ, 627, L89

Cooray A., 2006, MNRAS, 365, 842

Fontana A., et al., 2006, A\&A, 459, 745

Guo Y., et al., 2009, preprint, arXiv:0901.1150

Hansen S.M., Sheldon E.S., Wechsler R.H., Koester B.P., 2007, preprint (arXiv:0710.3780

Jing Y.P., Mo H.J., Börner G., 1998, ApJ, 494, 1

Jing Y.P., Börner G., Suto Y., 2002, ApJ, 564, 15

Kassin S.A., et al., 2007, ApJ, 660L, 35

Kroupa P., 2001, MNRAS, 322, 231

Li C., Kauffmann G., Jing Y.P., White S.D.M., Börner G., Cheng F.Z., 2006, MNRAS, 368, 21

Lin H., Kirshner R.P., Shectman S.A., Landy S.D., Oemler A., Tucker D.L., Schechter P.L., 1996, ApJ, 464, 60

Lin Y.T. Mohr J.J., 2004, ApJ, 617, 879

Ludlow Ä.D., Navarro J.F., Springel V., Jenkins A., Frenk C.S., Helmi A., 2008, preprint (arXiv:0801.1127)

Madgwick D.S., et al., 2002, MNRAS, 333, 133

McGaugh S.S., 2005, ApJ, 632, 859

McIntosh D.H., Guo Y., Hertzberg J., Katz N., Mo H.J., van den Bosch F.C., Yang X., 2007, preprint (arXiv:0710.2157)

Mo H.J., Yang X.H., van den Bosch F.C., Jing Y.P., 2004, MNRAS, 349, 205

More S. van den Bosch F.C., Cacciato M., Mo H.J., Yang X., Li R., 2008, MNRAS, submitted (arXiv:0807:4532)

Norberg P., et al., 2002, MNRAS, 336, 907

Panter B., Jimenez R., Heavens A. F., Charlot S., 2007, MNRAS, 378,1550

Peacock J.A., Smith R.E., 2000, MNRAS, 318, 1144

Petrosian V., 1976, ApJ, 209, L1

Pizagno J. et al., 2007, AJ, 134, 945
Popesso P., Biviano A., Böhringer H., Romaniello M., 2006, A\&A, 445, 29

Robotham A., Wallace C., Phillipps S., De Propris R., 2006, ApJ, 652,1077

Schlegel D.J., Finkbeiner D.P., Davis M., 1998, ApJ, 500, 525

Scoccimarro R., Sheth R.K., Hui L., Jain B., 2001, ÂpJ, 546, 20

Scranton R., 2002a, MNRAS, 332, 697

Seljak U.,2000, MNRAS, 318, 203

Sheldon E.S., et al., 2007, preprint, arXiv:0709.1162

Skibba R.A., Sheth R.K., Martino M.C., 2007, MNRAS, 382, 1940

Skibba R.A., Sheth R.K., 2008, preprint, arXiv:0805.0310

Skibba R.A., 2008, preprint, arXiv:0805.1233

Spergel, D. N., et al. 2007, ApJS, 170, 377

Stoughton C., et al., 2002, AJ, 123, 485

Strauss M.A., et al., 2002, AJ, 124, 1810

Tago E., et al., 2006. Astronomische Nachrichten, 327, 365

Tempel E., Einasto J., Einasto M., Saar E., 2008, preprint, arXiv:0805.4264

Tinker J.L., Weinberg D.H., Zheng Z., Zehavi I., 2005, ApJ, 631, 41

Tully R. B., Pierce M.J, 2000, ApJ, 533, 744

Vale A., Ostriker J.P., 2004, MNRAS, 353, 189

Vale A., Ostriker J.P., 2006, MNRAS, 371, 1173

Vale A., Ostriker J.P., 2007, preprint (astro-ph/0701096

van den Bosch F.C., Yang X.., Mo H.J., 2UU3, MINRÁs, 340, 771

van den Bosch F. C., Mo H.J, Yang X 2003, MNRAS, 345, 923

van den Bosch F.C., Weinmann S.M., Yang X., Mo H.J., Li C., Jing Y.P., 2005, MNRAS, 361, 1203

von der Linden A., Best P.N., Kauffmann G., White S.D.M., 2007, MNRAS, 379, 867

van den Bosch F.C., Yang X., Mo H.J., Weinmann S.M., Maccio

A., More S., Cacciato M., Skibba R., Kang X., 2007, MNRAS, 376,841

van den Bosch F.C., Aquino D., Yang X., Mo H.J., Pasquali A., McIntosh D.H., Weinmann S.M., Kang X., 2008, MNRAS, 387, 79

Wang Y., Yang X., Mo H.J., van den Bosch F.C., Chu Y., 2004, MNRAS, 353, 287

Wang Y., Yang X., Mo H.J., van den Bosch F.C., Weinmann S.M., Chu Y., 2008a, ApJ, 687, 919

Wang Y., Yang X., Mo H.J., van den Bosch F.C., Katz N., Pasquali A.. McIntosh D.H., Weinmann S.M., 2008b, preprint, arXiv:0812.3723

Warren M.S., Abazajian K., Holz D.E., Teodoro L., 2006, ApJ, 646,881

Weinmann S.M., van den Bosch F.C., Yang X., Mo H.J., 2006a, MNRAS, 366, 2

Weinmann S.M., van den Bosch F.C., Yang X., Mo H.J., Croton D.J., Moore, B., 2006b, MNRAS, 372, 1161

White M., Zheng Z., Brown M.J.I., Dey A., Jannuzi B.T., 2007, ApJ, 655, 69

Yan R., Madgwick D.S., White M., 2003, ApJ, 598, 848

Yan R., White M., Coil A.L., 2004, ApJ, 607, 739

Yang X., Mo H.J., van den Bosch F.C., 2003, MNRAS, 339, 1057

Yang X., Mo H.J., Jing Y.P., van den Bosch F.C., Chu Y.Q., 2004, MNRAS, 350, 1153

Yang X., Mo H.J., van den Bosch F.C., Jing Y.P., 2005a, MNRAS, 356, 1293

Yang X., Mo H.J., van den Bosch F.C., Jing Y.P., 2005b, MNRAS, 357,608 
Yang X., Mo H.J., Jing Y.P., van den Bosch F.C. 2005c, MNRAS, 358, 217 (Y05c)

Yang X., Mo H.J., van den Bosch F.C., Weinmann S.M., Li C., Jing Y.P., 2005d, MNRAS, 362, 711

Yang X., Mo H.J., van den Bosch F.C., 2006, ApJ, 638L, 55

Yang X., Mo H.J., van den Bosch F.C., Pasquali A., Li C.,

Barden M., 2007, ApJ, 671, 153 (Paper I)

Yang X., Mo H.J., van den Bosch F.C., 2008a, ApJ, 676, 248 (Paper II)

Yang X., Mo H.J., van den Bosch F.C., 2008b, preprint, arXiv:0808.2526
Zandivarez A., Martnez H.J., Merchán M.E., 2006, ApJ, 650, 137 Zehavi I., et al., 2002, ApJ, 571, 172

Zehavi I., et al., 2004 ApJ, 608, 16

Zehavi I., et al., 2005 ApJ, 630, 1

Zheng Z., et al., 2005, ApJ, 633, 791

Zheng Z., Coil A.L., Zehavi I., 2007, ApJ, 667, 760

Zheng Z., Weinberg D.H., 2007, ApJ, 659, 1 